\title{
Entanglement entropy of linearized gravitons in a sphere
}

\author{
Valentin Benedetti®* and Horacio Casini ${ }^{\dagger}$ \\ Centro Atómico Bariloche and CONICET, S.C. de Bariloche, Río Negro R8402AGP, Argentina
}

(Received 6 September 2019; accepted 24 January 2020; published 7 February 2020)

\begin{abstract}
We compute the entanglement entropy of a massless spin 2 field in a sphere in flat Minkowski space. We describe the theory with a linearized metric perturbation field $h_{\mu \nu}$ and decompose the field into independent modes using tensor spherical harmonics. We fix the gauge such that (a) the two dynamical modes for each angular momentum decouple and have the dynamics of scalar spherical modes, and (b) the gauge-fixed field degrees of freedom inside the sphere represent gauge invariant operators of the theory localized in the same region. In this way the entanglement entropy turns out to be equivalent to the one of a pair of free massless scalars where the contributions of the $l=0$ and $l=1$ modes have been subtracted. The result for the coefficient of the universal logarithmic term is $-61 / 45$ and coincides with that computed using a regularization based on the mutual information.
\end{abstract}

DOI: 10.1103/PhysRevD.101.045004

\section{INTRODUCTION}

The entanglement entropy (EE) of vacuum fluctuations across a boundary in space has shown to be an interesting theoretical quantity in quantum field theory (QFT). The study of EE was originally motivated by the quest to understand black hole entropy and entropy in gravity, but it turned out to have a more clear and natural formulation in QFT. Entropy in quantum mechanics is by definition a quantity associated with a state in an algebra of operators, and ordinary QFT naturally comes with a built-in correspondence of algebras with regions of the space.

The situation in gravity is less clear precisely because it is not completely understood how "regions" in quantum gravity might be defined in terms of the operator content of the theory (see for example [1,2]). Holographic theories give a simple, but perhaps only partial, answer, to this question. By restricting the region to a boundary region, the associated algebra is given by one of the dual QFTs in the boundary. Holographic EE [3,4] has shown there is a correspondence, at least at the semiclassical level, of this QFT entropy to an entropy in a gravity theory in the socalled entanglement wedge [5-7].

As a first step in trying to understand the contributions of gravitons to the entropy, in this paper, we compute the EE of free gravitons in flat space. We treat the theory as a

\footnotetext{
*valentin.benedetti@gmail.com

casini@cab.cnea.gov.ar
}

Published by the American Physical Society under the terms of the Creative Commons Attribution 4.0 International license. Further distribution of this work must maintain attribution to the author(s) and the published article's title, journal citation, and DOI. Funded by SCOAP. quantum field theory of helicity 2 particles. In this sense, the problem is conceptually simpler because we do not have to deal with the localization issues of a full quantum gravity theory. Indeed, one of the motivations of this paper is to show there are no conceptual problems for these helicity 2 free fields per se as QFT.

In the study of EE, it is important to establish a correspondence of the different terms on the entropy with known physical quantities in the model. One such signature that allows us to distinguish models from their EE is given by the coefficient of the logarithmic term. For the gravitons, we treat the case of a sphere, computing the universal logarithmic term. For a general CFT, the logarithmic term is proportional to the $A$-anomaly $[8,9]$. This is the quantity decreasing along the renormalization group in dimension $d=4$. For the graviton, the usual definition of the $A$ anomaly is not valid since there is no stress tensor. However, we show the logarithmic term in the entropy is perfectly well defined, and compute its universal coefficient. We expect this type of contribution to be part of the full graviton entropy in the semiclassical limit.

As in the case of the Maxwell field, it is important in computing the EE to understand correctly what is the entropy one is computing, that is, what is the algebra and the state, as well as the meaning of the result in terms of the continuum theory. A natural way to do this is by interpreting the universal coefficients in terms of mutual information. This is transparent in the real-time formulation that we use in this paper where we have the quantum degrees of freedom (d.o.f.) always in sight. Computations using the replica trick may actually hide the nature of the entropy one is computing in the precise definition of the replica partition functions [10].

In this sense, there are in the literature several calculations of logarithmic corrections to the black hole entropy formula 
due to the $\mathrm{EE}$ of quantum fields in the semiclassical background, including gravitons (for a review see $[11,12]$ ). The graviton contribution may be of relevance to distinguish the gravity theory [12]. Nevertheless, logarithmic terms are subtle too. An example of the problems involved is in the case of a Maxwell field. The logarithmic term for a free Maxwell field does not coincide with the expected trace anomaly [13-15]. However, the presence of electric or magnetic charges can change this result, no matter the mass of the charged particles $[10,16]$. This issue has been also discussed in the literature using the effective constructions of edge modes or extended Hilbert space (see for example $[17,18]$ ).

To compute the entanglement entropy, we should consider the vacuum state in the algebra of gauge invariant operators. This later is generated by the curvature tensor which is gauge invariant at the linearized level. The vacuum is a Gaussian state in this algebra and we could apply EE formulas for Gaussian states in terms of the correlation functions and commutators of the Gaussian variables. However, due to the algebraic complexity of dealing with the four index curvature tensor and its commutators, we will follow a different route that is physically equivalent and will allow us to simplify the computations considerably. We will use the metric perturbation tensor $h_{\mu \nu}$ as a generator of the algebra. This is not a physical variable and we need to fix the gauge. This is done by taking into account the spherical symmetry of the problem by choosing a gauge that allows us to decouple the two radial modes for each angular momentum. However, as explained in [19], while fixing the gauge converts a gauge field into a physical variable, the localization properties of these variables are very much gauge dependent. Hence we need to fix the gauge such that the gauge fixed $h_{\mu \nu}$ can be recovered from the curvature inside the region of interest for computing the EE. Otherwise, selecting the field and momentum variables in a region may compute the EE of an algebra unrelated to geometry.

Since this gauge fixing procedure adapted to the region of interest has not been explicitly carried out in the literature before, we find it instructive to see how this works in the simpler case of a Maxwell field first. We will treat the case of a Maxwell field between parallel planes in the next section and in a sphere in Sec. III. The results agree with [13] where the algebra was defined directly in terms of the electric and magnetic fields instead of the gauge fixed vector potential $A_{\mu}$. In Sec. IV we describe the theory of the linearized graviton and compute the EE between parallel planes. The case of a sphere is treated in Sec. V where we compute the logarithmic coefficient. We end with a discussion in Sec. VI, where we briefly compare with other results in the literature.

\section{ENTANGLEMENT ENTROPY OF A MAXWELL FIELD BETWEEN PARALLEL PLANES}

Before studying the problem of linearized gravitons, we consider the simpler case of a free Maxwell field. In $(3+1)$ dimensions, the corresponding Lagrangian reads

$$
\begin{aligned}
L & =-\frac{1}{4} \int d^{3} x F_{\mu \nu} F^{\mu \nu} \\
& =\frac{1}{2} \int d^{3} x\left[\left(\dot{\vec{A}}(\vec{x})+\nabla A_{0}(\vec{x})\right)^{2}-(\nabla \times \vec{A}(\vec{x}))^{2}\right] .
\end{aligned}
$$

In this section, we aim to obtain the EE associated with the region $V$ between two parallel planes separated by a distance $L$ (Fig. 1). In a Cartesian coordinate system $\vec{x}=\left(x^{1}, x^{2}, x^{3}\right)$, the region $V$ is given by $V=$ $\left\{x=\left(x^{1}, x^{2}, x^{3}\right), 0<x^{1}<L\right\}$. For this purpose, it is particularly useful to write the field $A_{\mu}$ on a plane wave basis using the Fourier sum over the directions parallel to the plates. Assuming that the directions $x^{2}$ and $x^{3}$ are compactified to large sizes $R_{2}$ and $R_{3}$ with periodic boundary conditions, we can obtain

$$
A_{\mu}\left(x^{0}, x^{1}, x^{2}, x^{3}\right)=\sum_{\vec{k}} N e^{i \vec{k} \cdot \vec{x}} A_{\mu}\left(x^{0}, x^{1}, k\right),
$$

where it is valid that $A_{\mu}^{\dagger}\left(x^{0}, x^{1}, k\right)=A_{\mu}\left(x^{0}, x^{1},-k\right)$ and $N$ takes the value $\left[\sqrt{2 \pi R_{2} R_{3}}\right]^{-1}$. Moreover, the vector $\vec{k}$ can be expressed for $n^{2}, n^{3}=0, \pm 1, \pm 2, \ldots, \pm \infty$ as

$$
\vec{k}=\left(0, \frac{2 \pi n^{2}}{R_{2}}, \frac{2 \pi n^{3}}{R_{3}}\right) .
$$

The problem then decomposes into independent $(1+1)$ dimensional fields, labeled by $\vec{k}$, in the directions $x^{0}$, and $x^{1}$. To study a fixed mode, we can use a coordinate system adapted to $\vec{k}$, where $\hat{x}^{2}=\hat{k}$ and $\hat{x}^{3}=\hat{x}^{1} \times \hat{k}$. In these

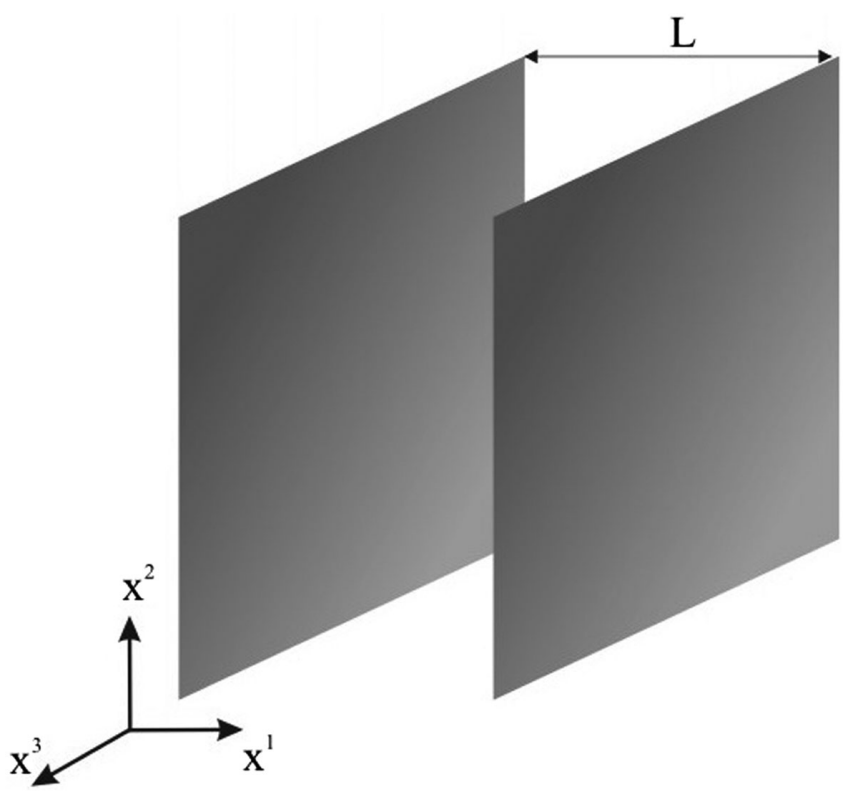

FIG. 1. Two parallel planes with a separation of distance $L$ in the $x^{1}$ direction. 
coordinates, the expressions that govern each mode take the form

$$
N e^{i k x_{2}} A_{\mu}\left(x_{0}, x_{1}, k\right)
$$

The gauge freedom of the Maxwell field is given by $A_{\mu}^{\prime} \rightarrow A_{\mu}+\partial_{\mu} \chi$. So, we also decompose $\chi$ in the plane wave basis. The mode corresponding to $\vec{k}$ reads

$$
\chi\left(x_{0}, x_{1}, x_{2}, x_{3}\right)=N e^{i k x_{2}} \chi\left(x_{0}, x_{1}, k\right) .
$$

Then, a gauge transformation of a fixed mode yields

$$
\begin{aligned}
A_{\mu}^{\prime}\left(x_{0}, x_{1}, x_{2}, x_{3}\right)= & N e^{i k x_{2}}\left[\left(A_{0}\left(x_{0}, x_{1}, k\right)+\dot{\chi}\left(x_{0}, x_{1}, k\right)\right) \hat{x}_{0}\right. \\
& +\left(A_{1}\left(x_{0}, x_{1}, k\right)+\partial_{1} \chi\left(x_{0}, x_{1}, k\right)\right) \hat{x}_{1} \\
& +\left(A_{2}\left(x_{0}, x_{1}, k\right)+i k \chi\left(x_{0}, x_{1}, k\right)\right) \hat{x}_{2} \\
& \left.+A_{3}\left(x_{0}, x_{1}, k\right) \hat{x}_{3}\right] .
\end{aligned}
$$

Now, it is clear that we can fix $\chi$ in such a way that the field components parallel to each $\hat{k}$ vanish. Using this choice of gauge, we can express $A_{\mu}$ as a local function of the tensor $F_{\mu \nu}$ in the coordinates $x^{0}$, and $x^{1}$

$$
F_{2 \nu}=\partial_{2} A_{\nu}-\partial_{\nu} A_{2}=i k A_{\nu}
$$

This allows us to identify the algebra of gauge invariant operators $F_{\mu \nu}$ in between the parallel planes with the one of the quantized gauge fixed operators $A_{\mu}$.

We aim to compute the Hamiltonian. To proceed, we must rewrite the Lagrangian (2.1) by using the expansion (2.4) under the proposed gauge condition. By doing so, we obtain for each mode the Lagrangian

$$
\begin{aligned}
\mathcal{L}_{k}= & 1 / 2\left[\dot{A}_{1}^{\dagger} \dot{A}_{1}+\dot{A}_{3}^{\dagger} \dot{A}_{3}-k^{2} A_{1}^{\dagger} A_{1}-k^{2} A_{3}^{\dagger} A_{3}-\partial_{1} A_{3}^{\dagger} \partial_{1} A_{3}\right. \\
& \left.-k^{2} A_{0}^{\dagger} A_{0}-\partial_{1} A_{0}^{\dagger} \partial_{1} A_{0}-A_{0}^{\dagger} \partial_{1} \dot{A}_{1}-\partial_{1} \dot{A}_{1}^{\dagger} A_{0}\right] .
\end{aligned}
$$

The canonical momenta of the fields $A_{1}, A_{1}^{\dagger}, A_{3}, A_{3}^{\dagger}$ are given by

$$
\begin{array}{ll}
\pi_{1}=\frac{\partial \mathcal{L}_{k}}{\partial \dot{A}_{1}}=\frac{\dot{A}_{1}^{\dagger}}{2}+\frac{\partial_{1} A_{0}^{\dagger}}{2}, & \pi_{3}=\frac{\partial \mathcal{L}_{k}}{\partial \dot{A}_{3}}=\frac{\dot{A}_{3}^{\dagger}}{2}, \\
\pi_{1}^{\dagger}=\frac{\partial \mathcal{L}_{k}}{\partial \dot{A}_{1}^{\dagger}}=\frac{\dot{A}_{1}}{2}+\frac{\partial_{1} A_{0}}{2}, & \pi_{3}^{\dagger}=\frac{\partial \mathcal{L}_{k}}{\partial \dot{A}_{3}^{\dagger}}=\frac{\dot{A}_{3}}{2} .
\end{array}
$$

The Hamiltonian of the mode is then given by the Legendre transform

$$
\begin{aligned}
\mathcal{H}_{k}= & \pi_{1} \dot{A}_{1}+\pi_{1}^{\dagger} \dot{A}_{1}^{\dagger}+\pi_{3} \dot{A}_{3}+\pi_{3}^{\dagger} \dot{A}_{3}^{\dagger}-\mathcal{L}_{k} \\
= & 2 \pi_{1}^{\dagger} \pi_{1}+2 \pi_{3}^{\dagger} \pi_{3}+\frac{k^{2}}{2} A_{1}^{\dagger} A_{1}+\frac{k^{2}}{2} A_{3}^{\dagger} A_{3}+\frac{1}{2} \partial_{1} A_{3}^{\dagger} \partial_{1} A_{3} \\
& +\frac{k^{2}}{2} A_{0}^{\dagger} A_{0}+A_{0}^{\dagger} \partial_{1} \pi_{1}^{\dagger}+A_{0} \partial_{1} \pi_{1}
\end{aligned}
$$

with the corresponding equal time commutation relations

$$
\begin{aligned}
& {\left[A_{1}\left(x_{0}, x_{1}, k\right), \pi_{1}\left(x_{0}, x_{1}^{\prime}, k\right)\right]=i \delta\left(x_{1}-x_{1}^{\prime}\right),} \\
& {\left[A_{3}\left(x_{0}, x_{1}, k\right), \pi_{3}\left(x_{0}, x_{1}^{\prime}, k\right)\right]=i \delta\left(x_{1}-x_{1}^{\prime}\right) .}
\end{aligned}
$$

It is clear from (2.11) that the field $A_{0}$ does not possess its own dynamic and thus it can be treated as a Lagrange multiplier. Differentiating, in order to obtain its equations of motion, we obtain the constraints

$$
\partial_{1} \pi_{1}=-\frac{k^{2}}{2} A_{0}^{\dagger}, \quad \partial_{1} \pi_{1}^{\dagger}=-\frac{k^{2}}{2} A_{0} .
$$

Replacement of (2.13) in (2.11) gives

$$
\begin{aligned}
\mathcal{H}_{k}= & 2 \pi_{1}^{\dagger} \pi_{1}+2 \pi_{3}^{\dagger} \pi_{3}+\frac{k^{2}}{2} A_{1}^{\dagger} A_{1}+\frac{k^{2}}{2} A_{3}^{\dagger} A_{3}+ \\
& +\frac{1}{2} \partial_{1} A_{3}^{\dagger} \partial_{1} A_{3}+\frac{2}{k^{2}} \partial_{1} \pi_{1} \partial_{1} \pi_{1}^{\dagger} .
\end{aligned}
$$

Making the identifications

$$
\begin{array}{ll}
\phi_{1}=\frac{\sqrt{2} \pi_{1}}{|k|}, & P_{1}=-\frac{|k| A_{1}}{\sqrt{2}}, \\
\phi_{3}=\frac{A_{3}}{\sqrt{2}}, & P_{3}=\sqrt{2} \pi_{3},
\end{array}
$$

where $\phi_{1}, P_{1}$ and $\phi_{3}, P_{3}$ are pairs of canonically conjugate variables, the Hamiltonian reads

$$
\begin{aligned}
\mathcal{H}_{k}= & P_{1}^{\dagger} P_{1}+P_{3}^{\dagger} P_{3}+\partial_{1} \phi_{1}^{\dagger} \partial_{1} \phi_{1}+\partial_{1} \phi_{3}^{\dagger} \partial_{1} \phi_{3} \\
& +k^{2} \phi_{1}^{\dagger} \phi_{1}+k^{2} \phi_{3}^{\dagger} \phi_{3} .
\end{aligned}
$$

This is exactly the Hamiltonian of the modes associated with two independent scalar fields that have been dimensionally reduced (see for example [13]). As a result, the algebra of gauge invariant operators of the gauge field inside the parallel planes is identical to the one corresponding to two massless scalar fields inside the same region. The same argument is valid for vacuum expectation values.

To sum up, we conclude that the EE of the Maxwell field associated with a region $V$ enclosed by two parallel planes is equivalent to one of two independent scalar fields. In this way, we recover the known result obtained in [13] by 
working with the gauge invariant electric and magnetic fields directly. The entropy turns out to be [20]

$$
S=c \frac{A}{\epsilon^{2}}-2 k_{s} \frac{A}{L^{2}},
$$

where $A=R_{2} R_{3}$ is the area of the planes, $\epsilon$ is a short distance cutoff, $c$ is a nonuniversal constant, and $k_{s}$ is the universal coefficient corresponding to a scalar in this same geometry. The later can be computed with high precision from the knowledge of the one dimensional scalar entropy function [21]. This calculation gives the result

$$
k_{s}=0.0055351599 \ldots
$$

As we will now see, this exact identification of entropies between scalars and gauge fields does not hold for other regions.

\section{ENTANGLEMENT ENTROPY FOR A MAXWELL FIELD IN THE SPHERE}

We consider now the problem of a Maxwell field on a sphere, which also can be easily dimensionally reduced. Due to the spherical symmetry presented in this case, we expand the field in question using scalar spherical harmonics for the $A_{0}$ component and vector spherical harmonics for $\vec{A}=\left(A_{1}, A_{2}, A_{3}\right)$. That is

$A_{0}=\sum_{l m} A_{l m}^{0}(t, r) Y_{l m}(\theta, \phi), \quad l=0,1, \ldots, \infty, \quad-l \leq m \leq l$,

$$
\vec{A}=\sum_{s l m} A_{l m}^{s}(t, r) \bar{Y}_{l m}^{s}(\theta, \phi), \quad l=0,1, \ldots, \infty, \quad-l \leq m \leq l,
$$$$
s=r, e, m,
$$

where $\bar{Y}_{l m}^{s}$ are the vector spherical harmonics defined by

$\bar{Y}_{l m}^{r}(\theta, \varphi)=Y_{l m}(\theta, \varphi) \hat{r}, \quad l \geq 0, \quad-l \leq m \leq l$,

$\bar{Y}_{l m}^{e}(\theta, \varphi)=\frac{r \nabla Y_{l m}(\theta, \varphi)}{\sqrt{l(l+1)}}, \quad l>0, \quad-l \leq m \leq l$,

$\bar{Y}_{l m}^{m}(\theta, \varphi)=\frac{\vec{r} \times \nabla Y_{l m}(\theta, \varphi)}{\sqrt{l(l+1)}}, \quad l>0, \quad-l \leq m \leq l$.

Considering the gauge transformations, it is useful to expand the function $\chi$ using scalar spherical harmonics as

$$
\chi=\sum_{l m} \chi_{l m}(t, r) Y_{l m}(\theta, \phi) .
$$

This gives the transformation law

$$
\vec{A}^{\prime}=\sum_{l m}\left(A_{l m}^{r}+\partial_{r} \chi_{l m}\right) \bar{Y}_{l m}^{r}+\left(A_{l m}^{e}+\frac{\chi_{l m}}{r}\right) \bar{Y}_{l m}^{e}+A_{l m}^{m} \bar{Y}_{l m}^{m} .
$$

We see that it is possible to fix $\chi_{l m}$ completely in such a way that the "electric" coefficient $A_{l m}^{\prime e}$ is identically zero for each angular momentum. This particular choice of gauge is convenient because of other reasons too. For each mode, it allows us to write

$$
F_{e \mu}=\left(e^{\nu} \partial_{\nu}\right) A_{\mu}+\left(\partial_{\mu} e^{\nu}\right) A_{\nu}
$$

where $e^{\mu}$ is the unit vector in the direction of $\bar{Y}_{l m}^{e}$, and $e^{\mu} \partial_{\mu}$ is the derivative in such direction. The expression (3.8) shows that, in this gauge, we can recover $A_{\mu}$ on the sphere by the knowledge of the components $F_{e \mu}$ of the gauge invariant field tensor on the same region. The relation between the gauge fixed $A_{\mu}$ and $F_{\mu \nu}$ is nonlocal in the angular directions. However, it allows the mapping of the variables $A_{\mu}$ at fixed $r$ to physical variables with the same radius. This is because the derivatives involved in (3.8) are tangential to the surface of the sphere. This is a particular case of the general situation studied in [19] where it was shown that a gauge fixing that respects the localization of d.o.f. in a region can be chosen in such a way that $A_{\mu}$ vanishes on the boundary of the region in a direction parallel to the boundary itself. In the present example, this direction is the one of the electric vector harmonics.

From this point, we proceed in the same way as in the case of parallel planes. In particular, a useful writing of the Lagrangian can be obtained by means of replacing (3.1) and (3.2) in (2.1). Then, by taking into consideration the orthonormality property of vector spherical harmonics, we get

$$
L=\sum_{l m} \int_{0}^{\infty} d r \mathcal{L}_{l m}
$$

The Lagrangian $\mathcal{L}_{l m}$ for $l \geq 1$ follows from direct computation using the properties of vector harmonics listed in Appendix A,

$$
\begin{aligned}
\mathcal{L}_{l m}= & 1 / 2\left[r^{2} \dot{A}_{l, m}^{r} \dot{A}_{l,-m}^{r}+r^{2} \dot{A}_{l, m}^{m} \dot{A}_{l,-m}^{m}-l(l+1) A_{l, m}^{r} A_{l,-m}^{r}\right. \\
& -l(l+1) A_{l, m}^{m} A_{l,-m}^{m}-\left|A_{l, m}^{m}+r \partial_{r} A_{l, m}^{m}\right|^{2} \\
& +r^{2} \partial_{r} A_{l, m}^{0} \partial_{r} A_{l,-m}^{0}+l(l+1) A_{l, m}^{0} A_{l,-m}^{0} \\
& -r^{2} A_{l, m}^{0} \partial_{r} \dot{A}_{l,-m}^{r}-r^{2} A_{l,-m}^{0} \partial_{r} \dot{A}_{l, m}^{r}+2 r A_{l, m}^{0} \dot{A}_{l,-m}^{r} \\
& \left.+2 r A_{l,-m}^{0} \dot{A}_{l, m}^{r}\right] .
\end{aligned}
$$

The Lagrangian density is independent of $m$. So, to simplify the notation in the following calculation we 
eliminate the index for $m$ in the variables and consider the real $m=0$ mode only. However, we should keep in mind that we will have $(2 l+1)$ identical contributions to the EE for each angular momentum. The canonical conjugate momenta are defined by

$\pi_{l}^{r}=\frac{\partial \mathcal{L}_{l}}{\partial \dot{A}_{l}^{r}}=r^{2}\left(\dot{A}_{l}^{r}+\partial_{r} A_{l}^{0}\right), \quad \pi_{l}^{m}=\frac{\partial \mathcal{L}_{l}}{\partial \dot{A}_{l}^{m}}=r^{2} \dot{A}_{l}^{m}$.

which can be substituted in the Legendre transform

$$
\mathcal{H}_{l}=\pi_{l}^{r} \dot{A}_{l}^{r}+\pi_{l}^{m} \dot{A}_{l}^{m}-\mathcal{L}_{l},
$$

in order to obtain the Hamiltonian

$$
\begin{aligned}
\mathcal{H}_{l}= & \frac{\pi_{l}^{r} \pi_{l}^{r}}{2 r^{2}}+\frac{\pi_{l}^{m} \pi_{l}^{m}}{2 r^{2}}+l(l+1) A_{l}^{r} A_{l}^{r}+l(l+1) A_{l}^{m} A_{l}^{m} \\
& +\left(A_{l}^{m}+r \partial_{r} A_{l}^{m}\right)^{2}-\pi_{l}^{r} \partial_{r} A_{l}^{0}-\frac{l(l+1)}{2} A_{l}^{0} A_{l}^{0} .
\end{aligned}
$$

The modes with different angular momentum are independent of each other and their operators commute. For a fixed $l$, the nontrivial canonical commutation relations are given by the following expression:

$$
\left[A_{l}^{r}(t, r), \pi_{l}^{r}\left(t, r^{\prime}\right)\right]=\left[A_{l}^{m}(t, r), \pi_{l}^{m}\left(t, r^{\prime}\right)\right]=i \delta\left(r-r^{\prime}\right) .
$$

Again, $A_{l}^{0}$ is a Lagrange multiplier, allowing the derivation of the constraint

$$
\partial_{r} \pi_{l}^{r}=l(l+1) A_{l}^{0},
$$

which can be replaced in (3.13) yielding

$$
\begin{aligned}
\mathcal{H}_{l}= & \frac{1}{2}\left[\frac{\pi_{l}^{r} \pi_{l}^{r}}{r^{2}}+\frac{\partial_{r} \pi_{l}^{r} \partial_{r} \pi_{l}^{r}}{l(l+1)}+l(l+1) A_{l}^{r} A_{l}^{r}\right] \\
& +\frac{1}{2}\left[\frac{\pi_{l}^{m} \pi_{l}^{m}}{r^{2}}+2\left(A_{l}^{m}+r \partial_{r} A_{l}^{m}\right)^{2}+l(l+1) A_{l}^{m} A_{l}^{m}\right] .
\end{aligned}
$$

Lastly, the field and momentum variables can be rewritten as

$$
\begin{aligned}
& \phi_{l}^{r}=\frac{\pi_{l}^{r}}{\sqrt{l(l+1)}}, \quad P_{l}^{r}=-\sqrt{l(l+1)} A_{l}^{r}, \\
& \phi_{l}^{m}=r A_{l}^{r}, \quad P_{l}^{m}=\frac{\pi_{l}^{m}}{r},
\end{aligned}
$$

and by applying (3.17) and (3.18) in (3.16) we reduce the Hamiltonian to the one of two identical radial modes given by

$$
\begin{aligned}
\mathcal{H}_{l}= & \frac{1}{2}\left[P_{l}^{r} P_{l}^{r}+\partial_{r} \phi_{l}^{r} \partial_{r} \phi_{l}^{r}+\frac{l(l+1)}{r^{2}} \phi_{l}^{r} \phi_{l}^{r}\right] \\
& +\frac{1}{2}\left[P_{l}^{m} P_{l}^{m}+\partial_{r} \phi_{l}^{m} \partial_{r} \phi_{l}^{m}+\frac{l(l+1)}{r^{2}} \phi_{l}^{m} \phi_{l}^{m}\right],
\end{aligned}
$$

with the standard commutation relations

$$
\begin{aligned}
{\left[\phi_{l}^{r}(t, r), P_{l}^{r}\left(t, r^{\prime}\right)\right] } & =\left[\phi_{l}^{m}(t, r), P_{l}^{m}\left(t, r^{\prime}\right)\right] \\
& =i \delta\left(r-r^{\prime}\right)
\end{aligned}
$$

Each of these two identical modes has the same Hamiltonian as the one that results from the spherical reduction of a free massless scalar field [13,22].

Equation (3.10) does not apply to the zero angular momentum mode. This is simply because the electric (3.4) and magnetic (3.5) spherical harmonics do not exist for $l=0$. For $l=0$ we get the simpler expression

$$
\mathcal{H}_{0}=\frac{\pi_{0}^{r} \pi_{0}^{r}}{2 r^{2}}+A_{0}^{0} \partial_{r} \pi_{0}^{r}
$$

where by replacing the constraint $\partial_{r} \pi_{l=0}^{r}=0$, obtained from the equations of motion of $A_{0}^{0}$, we get

$$
\mathcal{H}_{0}=\frac{\pi_{0}^{r} \pi_{0}^{r}}{2 r^{2}}
$$

This means that the zero angular momentum mode does not have dynamics and thus generates no contributions to the entropy.

Therefore, the EE of the Maxwell field on the sphere is equivalent to the one of two scalar fields where the $l=0$ mode has been subtracted. This result coincides with the one given in [13]. The entanglement entropy of a scalar in a sphere has a universal logarithmic term $-1 / 90 \log (R / \epsilon)$ $[8,9,14,23,24]$. The mode $l=0$ for the scalar [see the Hamiltonian (3.19) for $l=0$ ] corresponds to a massless $d=2$ field in the $r>0$ half-line with Dirichlet boundary condition at the origin, and its universal logarithmic entropy is $1 / 6 \log (R / \epsilon) \quad[13,25]$. The entropy of the Maxwell field in the sphere is then given by $[13,14]$

$$
S=c \frac{A}{\epsilon^{2}}-\frac{16}{45} \log \left(\frac{R}{\epsilon}\right),
$$

where the coefficient of the logarithmic term follows from $16 / 45=2 \times 1 / 90+2 \times 1 / 6$.

Again, we recover this result by working with the gauge variant field $A_{\mu}$ instead of using directly the electric and magnetic gauge invariant fields as in [13]. It is important to remark that another gauge choice that does not respect the 
locality on the sphere would have given a completely different and incorrect result for the sphere EE.

\section{ENTANGLEMENT ENTROPY OF LINEARIZED GRAVITONS BETWEEN PARALLEL PLANES}

The free theory of a massless helicity 2 particle can be described by a field $h_{\mu \nu}$. This field can be thought of as describing metric perturbations $g_{\mu \nu}=\eta_{\mu \nu}+h_{\mu \nu}$ with respect to the Minkowski metric $\eta_{\mu \nu}$. The field $h_{\mu \nu}$ obeys the linearized Einstein equations and the Lagrangian that gives these equations in absence of sources reads [26]

$$
\begin{aligned}
\mathcal{L}= & -\partial_{\mu} h^{\mu \nu} \partial_{\alpha} h^{\alpha}{ }_{\nu}+\frac{1}{2} \partial^{\alpha} h_{\mu \nu} \partial_{\alpha} h^{\mu \nu}+\partial_{\mu} h^{\mu \nu} \partial_{\nu} h^{\alpha}{ }_{\alpha} \\
& -\frac{1}{2} \partial_{\alpha} h^{\mu}{ }_{\mu} \partial^{\alpha} h^{\nu}{ }_{\nu} .
\end{aligned}
$$

The theory has a gauge invariance given by the transformation law

$$
h_{\mu \nu}^{\prime}=h_{\mu \nu}+\partial_{\nu} \xi_{\mu}+\partial_{\mu} \xi_{\nu}
$$

for an arbitrary vector field $\xi_{\mu}$. This corresponds to the diffeomorphism invariance of the Einstein theory of gravity at the linearized level.

The curvature is not gauge invariant in the nonlinear gravity theory. However, a gauge invariant operator corresponds to the linearized curvature tensor [27]
$R_{\mu \nu \rho \sigma}=\frac{1}{2}\left[\partial_{\nu} \partial_{\rho} h_{\mu \sigma}-\partial_{\mu} \partial_{\rho} h_{\nu \sigma}+\partial_{\mu} \partial_{\theta} h_{\nu \rho}-\partial_{\nu} \partial_{\sigma} h_{\mu \rho}\right]$.

It is a simple exercise to show that it is indeed invariant under the transformations (4.2). ${ }^{1}$ Therefore, in contrast to what is expected in full quantum gravity, the theory of a helicity 2 field in Minkowski space contains gauge invariant local operators. In consequence, the EE is well defined, except for the usual issues about divergent terms. As a first exercise, let us study the case of a region bounded by two parallel planes.

\section{A. Plane wave decomposition and gauge fixing}

For the wall between parallel planes, we resort to a plane wave decomposition of the fields analogous to (2.4). We write the field of a mode with $\vec{k}=k \hat{x}_{2}$ as

$$
h_{\mu \nu}\left(x_{0}, x_{1}, x_{2}, x_{3}\right)=N e^{i k x_{2}} h_{\mu \nu}\left(x_{0}, x_{1}, k\right) .
$$

The arbitrary gauge function $\xi$ can also be decomposed in modes. The mode associated with a vector $\vec{k}$ reads

$$
\xi_{\mu}\left(x_{0}, x_{1}, x_{2}, x_{3}\right)=N e^{i k x_{2}} \xi_{\mu}\left(x_{0}, x_{1}, k\right) .
$$

Therefore, it can be easily observed that linearized diffeomorphisms give the following transformation law:

$$
h_{\mu \nu}^{\prime}=\left[\begin{array}{cccc}
h_{00}+2 \dot{\xi}_{0} & h_{01}+\dot{\xi}_{1}+\partial_{1} \xi_{0} & h_{02}+\dot{\xi}_{2}+i k \xi_{0} & h_{03}+\dot{\xi}_{3} \\
h_{01}+\dot{\xi}_{1}+\partial_{1} \xi_{0} & h_{11}+2 \partial_{1} \xi_{1} & h_{12}+\partial_{1} \xi_{2}+i k \xi_{2} & h_{13}+\partial_{1} \xi_{3} \\
h_{02}+\dot{\xi}_{2}+i k \xi_{0} & h_{12}+\partial_{1} \xi_{2}+i k \xi_{2} & h_{22}+2 i k \xi_{2} & h_{23}+i k \xi_{3} \\
h_{03}+\dot{\xi}_{3} & h_{13}+\partial_{1} \xi_{3} & h_{23}+i k \xi_{3} & h_{33}
\end{array}\right] .
$$

Now, it is clear that the components $h_{02}^{\prime}, h_{20}^{\prime}, h_{12}^{\prime}, h_{21}^{\prime}, h_{22}^{\prime}$, $h_{23}^{\prime}$, and $h_{32}^{\prime}$ can be fixed to zero if we use up all the available gauge freedom. All the components of $h_{\mu \nu}^{\prime}$ that have nonzero contractions in the direction of $\hat{k}$ are set to zero. For each mode, this allows us to write the $R_{2 \mu 2 \nu}$ component of the Riemann tensor as

$2 R_{2 \mu 2 \nu}=h_{\nu 2, \mu 2}+h_{2 \mu}, 2 \nu-h_{\nu \mu}, 22-h_{22, \mu \nu}=-h_{\nu \mu}, 22=k^{2} h_{\nu \mu}$.

\footnotetext{
${ }^{1}$ This corresponds to the fact that the curvature transforms linearly under changes of coordinates and it is already of linear order in $h_{\mu \nu}$. Then further factors of the infinitesimal coordinate transformation must be second order.
}

That is, under this particular choice of gauge, it is possible to write the field $h_{\nu \mu}$ in terms of the Riemann tensor in a local way in the $x_{0}$ and $x_{1}$ coordinates. This means that the algebra of the gauge fixed d.o.f. of $h_{\nu \mu}$ is the same as the algebra of the curvature tensor in between the planes.

\section{B. Lagrangian for each momentum}

The Lagrangian decomposes into independent modes for the different plane waves. For this purpose, we use the expansion (4.4) and the gauge condition presented in the previous section. The Lagrangian density for the $(1+1)$ dimensional theory of each mode $\vec{k}$ can be expressed as 


$$
\begin{aligned}
\mathcal{L}_{k}= & \dot{h}_{13} \dot{h}_{13}^{\dagger}-\frac{1}{2}\left(\dot{h}_{11} \dot{h}_{33}^{\dagger}+\dot{h}_{33} \dot{h}_{11}^{\dagger}\right)-k^{2} h_{13} h_{13}^{\dagger} \\
& +\frac{k^{2}}{2}\left(h_{11} h_{33}^{\dagger}+h_{33} h_{11}^{\dagger}\right)-\frac{k^{2}}{2} h_{00}\left(h_{11}^{\dagger}+h_{33}^{\dagger}\right) \\
& -\frac{k^{2}}{2}\left(h_{11}+h_{33}\right) h_{00}^{\dagger}-h_{01} \partial_{1} \dot{h}_{33}^{\dagger}-\partial_{1} \dot{h}_{33} h_{01}^{\dagger}+h_{03} \partial_{1} \dot{h}_{13}^{\dagger} \\
& +\partial_{1} \dot{h}_{13} h_{03}^{\dagger}+k^{2} h_{03} h_{03}^{\dagger}+k^{2} h_{01} h_{01}^{\dagger}+\partial_{1} h_{03} \partial_{1} h_{03}^{\dagger} \\
& -\frac{1}{2} \partial_{1} h_{33} \partial_{1} h_{00}^{\dagger}-\frac{1}{2} \partial_{1} h_{00} \partial_{1} h_{33}^{\dagger} .
\end{aligned}
$$

This Lagrangian contains two sets of independent fields. Then, the problem can be split into two modes that will be treated separately. The first one contains the field $h_{13}$ and the Lagrange multiplier $h_{03}$

$$
\begin{aligned}
\mathcal{L}_{I}= & \dot{h}_{13} \dot{h}_{13}^{\dagger}-k^{2} h_{13} h_{13}^{\dagger}-\partial_{1} h_{03} \dot{h}_{13}^{\dagger}-\dot{h}_{13} \partial_{1} h_{03}^{\dagger} \\
& +k^{2} h_{03} h_{03}^{\dagger}+\partial_{1} h_{03} \partial_{1} h_{03}^{\dagger},
\end{aligned}
$$

and the second one contains the fields $h_{11}$ and $h_{33}$ and the multipliers $h_{01}$ and $h_{00}$

$$
\begin{aligned}
\mathcal{L}_{I I}= & -\frac{1}{2}\left(\dot{h}_{11} \dot{h}_{33}^{\dagger}+\dot{h}_{33} \dot{h}_{11}^{\dagger}\right)+\frac{k^{2}}{2}\left(h_{11} h_{33}^{\dagger}+h_{33} h_{11}^{\dagger}\right) \\
& +\partial_{1} h_{01} \dot{h}_{33}^{\dagger}+\dot{h}_{33} \partial_{1} h_{01}^{\dagger}-\frac{k^{2}}{2} h_{00}\left(h_{11}^{\dagger}+h_{33}^{\dagger}\right) \\
& -\frac{k^{2}}{2}\left(h_{11}+h_{33}\right) h_{00}^{\dagger}+k^{2} h_{01} h_{01}^{\dagger}-\frac{1}{2} \partial_{1} h_{33} \partial_{1} h_{00}^{\dagger} \\
& -\frac{1}{2} \partial_{1} h_{00} \partial_{1} h_{33}^{\dagger} .
\end{aligned}
$$

Therefore, the total Lagrangian is given by sum over modes

$$
L=\sum_{k} \int_{0}^{\infty} d x_{1}\left(\mathcal{L}_{I}+\mathcal{L}_{I I}\right)
$$

\section{Hamiltonian of the mode I}

The momenta $\pi_{13}, \pi_{13}^{\dagger}$ corresponding to the Lagrangian (4.9) are

$$
\begin{aligned}
& \pi_{13}=\frac{\partial \mathcal{L}_{I}}{\partial \dot{h}_{13}}=\dot{h}_{13}^{\dagger}-\partial_{1} h_{03}^{\dagger}, \\
& \pi_{13}^{\dagger}=\frac{\partial \mathcal{L}_{I}}{\partial \dot{h}_{13}^{\dagger}}=\dot{h}_{13}-\partial_{1} h_{03},
\end{aligned}
$$

and the corresponding Hamiltonian is

$$
\begin{aligned}
\mathcal{H}_{I}= & \pi_{13} \dot{h}_{13}+\pi_{13}^{\dagger} \dot{h}_{13}^{\dagger}-\mathcal{L}_{I} \\
= & \pi_{13} \pi_{13}^{\dagger}+k^{2} h_{13} h_{13}^{\dagger}-h_{03} \partial_{1} \pi_{13}-h_{03}^{\dagger} \partial_{1} \pi_{13}^{\dagger} \\
& -k^{2} h_{03} h_{03}^{\dagger} .
\end{aligned}
$$

A constraint equation can be derived by computing the equation of motion of $h_{03}$

$$
h_{03}=\frac{\partial_{1} \pi_{13}^{\dagger}}{k^{2}}, \quad h_{03}^{\dagger}=\frac{\partial_{1} \pi_{13}}{k^{2}},
$$

which can be replaced in (4.13) to obtain

$$
\mathcal{H}_{I}=\pi_{13} \pi_{13}^{\dagger}+k^{2} h_{13} h_{13}^{\dagger}+\frac{\partial_{1} \pi_{13} \partial_{1} \pi_{13}^{\dagger}}{k^{2}} .
$$

In order to rewrite the Hamiltonian (4.15) as the one associated with a complex scalar (for each pair $\vec{k},-\vec{k}$ ), it is convenient to define

$$
\begin{array}{ll}
\phi_{I}=\frac{\pi_{13}}{|k|}, & P_{I}=-|k| h_{13}, \\
\phi_{I}^{\dagger}=\frac{\pi_{13}^{\dagger}}{|k|}, & P_{I}^{\dagger}=-|k| h_{13}^{\dagger} .
\end{array}
$$

In this way, (4.15) gets rewritten as

$$
\mathcal{H}_{I}=P_{1} P_{1}^{\dagger}+k^{2} \phi_{1} \phi_{1}^{\dagger}+\partial_{1} \phi_{1} \partial_{1} \phi_{1}^{\dagger} .
$$

Moreover, by canonical quantization of the field $h_{13}$ in (4.13), it is clear that the replacements (4.16) and (4.17) give $P_{1}$ and $\phi_{1}$ as canonically conjugate variables.

\section{Hamiltonian of the mode II}

Analyzing the dynamics of the fields $h_{00}$ and $h_{00}^{\dagger}$ in the Lagrangian (4.11), it is evident that both play the role of Lagrange multipliers. These allow us to derive the constraints

$$
\begin{aligned}
& \partial_{1} \partial_{1} h_{33}=k^{2}\left(h_{11}+h_{33}\right), \\
& \partial_{1} \partial_{1} h_{33}^{\dagger}=k^{2}\left(h_{11}^{\dagger}+h_{33}^{\dagger}\right) .
\end{aligned}
$$

If we replace (4.19) in (4.11) it is possible to eliminate the field $h_{11}$ from the Lagrangian, obtaining

$$
\begin{aligned}
\mathcal{L}_{I I}= & \dot{h}_{33} \dot{h}_{33}^{\dagger}-k^{2} h_{33} h_{33}^{\dagger}+\frac{\partial_{1} \dot{h}_{33} \partial_{1} \dot{h}_{33}^{\dagger}}{k^{2}} \\
& -\partial_{1} h_{33} \partial_{1} h_{33}^{\dagger}-h_{01} \partial_{1} \dot{h}_{33}^{\dagger}-\partial_{1} \dot{h}_{33} h_{01}^{\dagger} \\
& +k^{2} h_{01} h_{01}^{\dagger} .
\end{aligned}
$$

The substitution produced a higher derivative term $k^{-2} \partial_{1} \dot{h_{33}} \partial_{1} \dot{h_{33}}{ }^{\dagger}$. However, this problem disappears when we use the constraint equation related to the Lagrange multipliers $h_{01}$ and $h_{01}^{\dagger}$. Indeed, we obtain

$$
h_{01}=\frac{\partial_{1} \dot{h}_{33}}{k^{2}}, \quad h_{01}^{\dagger}=\frac{\partial_{1} \dot{h}_{33}^{\dagger}}{k^{2}} .
$$


By replacing (4.21) in (4.20) the Lagrangian gets reduced to

$$
\mathcal{L}_{I I}=\dot{h}_{33} \dot{h}_{33}^{\dagger}-k^{2} h_{33} h_{33}^{\dagger}-\partial_{1} h_{33} \partial_{1} h_{33}^{\dagger} .
$$

Then, the canonical momenta associated to the complex field variables $h_{33}$ and $h_{33}^{\dagger}$ are

$$
\pi_{33}=\frac{\partial \mathcal{L}_{I I}}{\partial \dot{h}_{33}}=\dot{h}_{33}^{\dagger}, \quad \pi_{33}^{\dagger}=\frac{\partial \mathcal{L}_{I I}}{\partial \dot{h}_{33}^{\dagger}}=\dot{h}_{33},
$$

and the corresponding Hamiltonian gets the same form as (4.18),

$$
\mathcal{H}_{I I}=P_{I I} P_{I I}^{\dagger}+k^{2} \phi_{I I} \phi_{I I}^{\dagger}+\partial_{1} \phi_{I I} \partial_{1} \phi_{I I}^{\dagger},
$$

where we have introduced the trivial notation change

$$
\begin{array}{ll}
\phi_{I I}=h_{33}, & P_{I I}=\pi_{13}, \\
\phi_{I I}^{\dagger}=h_{33}^{\dagger}, & P_{I I}^{\dagger}=\pi_{13}^{\dagger} .
\end{array}
$$

These variables also obey canonical commutation relations.

\section{E. Entanglement Entropy}

The Hamiltonians for the two modes (4.18) and (4.24) are equivalent to the ones of a dimensionally reduced scalar field. We are allowed to conclude that the EE of linearized gravitons for the region enclosed between two parallel planes is equivalent to the one of two scalar fields or one Maxwell field. The universal coefficients will be the same in the three cases. We get again

$$
S=c \frac{A}{\epsilon^{2}}-2 k_{s} \frac{A}{L^{2}},
$$

with $k_{s}$ given by (2.19) and [20].

\section{ENTANGLEMENT ENTROPY OF LINEARIZED GRAVITONS IN A SPHERE}

In this section, we treat the case of gravitons inside a sphere. We first introduce the tensor spherical harmonics that we use to decompose $h_{\mu \nu}$ in spherical coordinates. We also decompose the gauge transformations and choose a generic gauge adapted to the spherical symmetry that depends on three arbitrary constants. Then, we expand the Lagrangian in terms of the gauge fixed field to get two independent radial modes for each angular momentum. The gauge choice is further refined to allow the simplification of the mode Hamiltonians. In this context, we ensure the locality in the radial direction in the relation between the gauge fixed field and the curvature tensor. We get a system of modes that are equivalent to the scalar spherical modes except that the $l=0,1$ modes are absent. Finally, we compute the entanglement entropy.

\section{A. Tensor spherical harmonics}

The tensor spherical harmonics are a further generalization of the concept of scalar and vector spherical harmonics. They can be used as a basis for the space of symmetric tensors (of dimension six). An arbitrary symmetric tensor field $X$ can be expanded in polar coordinates as follows:

$$
\begin{aligned}
X= & \sum_{J s l m} X_{l m}^{J s}(r) T_{l m}^{J s}(\theta, \phi), \quad l=0,1, \ldots, \infty \\
& m=0, \pm 1, \ldots, \pm l, \quad J s=0 l, 0 t, 1 e, 1 m, 2 e, 2 m
\end{aligned}
$$

where the tensor spherical harmonics $T_{l m}^{J S}$ are given by (see for example $[28,29])$

$$
\begin{aligned}
& T_{l m}^{0 l}=\hat{r} \otimes \hat{r} Y_{l m}, \quad T_{l m}^{0 t}=\frac{1}{\sqrt{2}}(\delta-\hat{r} \otimes \hat{r}) Y_{l m}, \\
& T_{l m}^{1 e}=\sqrt{\frac{2}{l(l+1)} r\left[\hat{r} \otimes \nabla Y_{l m}\right]^{S},} \\
& T_{l m}^{1 m}=\sqrt{\frac{2}{l(l+1)}\left[\hat{r} \otimes \bar{r} \times \nabla Y_{l m}\right]^{S},} \\
& T_{l m}^{2 e}=\sqrt{2 \frac{(l-2) !}{(l+2) !}}\left[r^{2} \nabla \nabla Y_{l m}\right]^{S T T}, \\
& T_{l m}^{2 m}=\sqrt{2 \frac{(l-2) !}{(l+2) !}}\left[r \nabla\left(\bar{r} \times \nabla Y_{l m}\right)\right]^{S T T} .
\end{aligned}
$$

The spherical harmonics of spin $J=0$ are defined for $l \geq 0$, the ones related to $\operatorname{spin} J=1$ for $l \geq 1$, and the in the case of spin $J=2$ for $l \geq 2$. In the notation of Eq. (5.2), the symbol $\delta$ means the identity tensor $\delta_{i j}$. Additionally, the superscript $S$ means taking the symmetric part, and $T T$ the traceless part transverse to $\hat{r}$. For an arbitrary tensor $X_{i j}$ this later is given by the following expression:

$$
\begin{aligned}
X_{i j}^{T T}= & \left(\delta_{i k}-\hat{r}_{i} \hat{r}_{k}\right)\left(\delta_{j n}-\hat{r}_{j} \hat{r}_{n}\right) X_{k n} \\
& -\frac{1}{2}\left(\delta_{i j}-\hat{r}_{i} \hat{r}_{j}\right)\left[\left(\delta_{k n}-\hat{r}_{k} \hat{r}_{n}\right) X_{n k}\right] .
\end{aligned}
$$

It will be useful to have a relation between tensor and vector spherical harmonics. This relation can be expressed as 
$T_{l m}^{0 l}=\left[\hat{r} \otimes \bar{Y}_{l m}^{r}\right]^{S}, \quad T_{l m}^{0 t}=\frac{1}{\sqrt{2}}\left(\delta Y_{l m}-\hat{r} \otimes \bar{Y}_{l m}^{r}\right)$,

$T_{l m}^{1 e}=\sqrt{2}\left[\hat{r} \otimes \bar{Y}_{l m}^{e}\right]^{S}, \quad T_{l m}^{1 m}=\sqrt{2}\left[\hat{r} \otimes \bar{Y}_{l m}^{m}\right]^{S}$,

$T_{l m}^{2 e}=\sqrt{\frac{2}{(l-1)(l+2)}}\left\{\left[r \nabla \bar{Y}_{l m}^{e}\right]^{S}+\frac{1}{\sqrt{2}} T_{l m}^{1 e}+\sqrt{\frac{l(l+1)}{2}} T_{l m}^{0 t}\right\}$,

$T_{l m}^{2 m}=\sqrt{\frac{2}{(l-1)(l+2)}}\left\{\left[r \nabla \bar{Y}_{l m}^{m}\right]^{S}+\frac{1}{\sqrt{2}} T_{l m}^{1 m}\right\}$.

Further properties of the tensor spherical harmonics are listed in Appendix B.

\section{B. Decomposition of the spin 2 field in spherical harmonics}

To make the process of computing the EE easier, it will be useful to decompose the field $h_{\mu \nu}$ and the gauge arbitrary function $\xi_{\mu}$ on a basis adapted to spherical symmetry. For this purpose, we introduce the notation

$$
\begin{aligned}
h_{T} & =\left[\begin{array}{lll}
h_{11} & h_{12} & h_{13} \\
h_{21} & h_{22} & h_{23} \\
h_{31} & h_{32} & h_{33}
\end{array}\right], \quad h_{V}=\left[\begin{array}{l}
h_{01} \\
h_{02} \\
h_{03}
\end{array}\right], \quad h_{S}=h_{00}, \\
\xi_{V} & =\left[\begin{array}{l}
\xi_{1} \\
\xi_{2} \\
\xi_{3}
\end{array}\right], \quad \xi_{S}=\xi_{0} .
\end{aligned}
$$

Firstly, we will expand $h_{T}$ in tensor spherical harmonics and $\xi_{V}$ in vector spherical harmonics. Then, we will study the remaining gauge freedom by using vector spherical harmonics for $h_{V}$ and scalar spherical harmonics for $\xi_{S}$ and $h_{S}{ }^{2}$

\section{Gauge fixing for spacelike components}

As we just mentioned, $h_{T}$ and $\xi_{V}$ will be expanded using tensor and vector spherical harmonics respectively. We get the following expressions:

$$
h_{T}=\sum_{J s l m} h_{l m}^{J s}(t, r) T_{l m}^{J s}(\theta, \varphi), \quad \xi_{V}=\sum_{s l m} \xi_{l m}^{s}(t, r) \bar{Y}_{l m}^{s}(\theta, \varphi) .
$$

On the other hand, the gauge freedom of linear gravity can be expressed in this notation as

$$
h_{T}^{\prime}=h_{T}+\nabla \xi_{V}+\left[\nabla \xi_{V}\right]^{T}=h_{T}+2\left[\nabla \xi_{V}\right]^{S} .
$$

The combination of (5.6) and (5.7) gives

$h_{T}^{\prime}=\sum_{J s l m} h_{l m}^{J s} T_{l m}^{J s}+2 \sum_{s l m}\left[\xi_{l m}^{s} \nabla \bar{Y}_{l m}^{s}+\bar{Y}_{l m}^{s} \otimes \partial_{r} \xi_{l m}^{s} \hat{r}\right]^{S}$.

By computing $\xi_{l m}^{s} \nabla \bar{Y}_{l m}^{s}+\bar{Y}_{l m}^{s} \otimes \partial_{r} \xi_{l m}^{s} \hat{r} \quad$ using the properties of vector and tensor spherical harmonics (Appendixes A and $\mathrm{B}$ ), for $s=r, e, m$ separately, and then adding up these contributions we get

$$
\begin{aligned}
h_{T}^{\prime}= & \sum_{l m}\left(h_{l m}^{0 l}+2 \partial_{r} \xi_{l m}^{r}\right) T_{l m}^{0 l}+\left(h_{l m}^{0 t}+\frac{2 \sqrt{2}}{r} \xi_{l m}^{r}-\frac{\sqrt{2 l(l+1)}}{r} \xi_{l m}^{e}\right) T_{l m}^{0 t} \\
& +\left(h_{l m}^{1 e}+\frac{\sqrt{2 l(l+1)}}{r} \xi_{l m}^{r}+\sqrt{2} \partial_{r} \xi_{l m}^{e}-\frac{\sqrt{2}}{r} \xi_{l m}^{e}\right) T_{l m}^{1 e}+\left(h_{l m}^{1 m}+\sqrt{2} \partial_{r} \xi_{l m}^{m}-\frac{\sqrt{2}}{r} \xi_{l m}^{m}\right) T_{l m}^{1 m} \\
& +\left(h_{l m}^{2 e}+\frac{\sqrt{2(l-1)(l+2)}}{r} \xi_{l m}^{e}\right) T_{l m}^{2 e}+\left(h_{l m}^{2 m}+\frac{\sqrt{2(l-1)(l+2)}}{r} \xi_{l m}^{m}\right) T_{l m}^{2 m} .
\end{aligned}
$$

This particular case differs from the ones studied earlier because there are many possible reasonable choices of gauge fixing for the spherical waves. Not all of them will allow us to calculate the $\mathrm{EE}$ that corresponds to the spherical boundary or allow us to decouple the two dynamical modes for each $\mathrm{lm}$. More specifically, the following can be seen:

(1) Fixing $\xi_{r}$ allows us to cancel the components that are parallel to $T_{l m}^{0 t}$ or $T_{l m}^{1 e}$ or to a linear combination of them.

\footnotetext{
${ }^{2}$ See $[28,30]$ for a different but somewhat analogous treatment of gravitons in spherical coordinates.
}

(2) Fixing $\xi_{e}$ allows us to cancel the components that are parallel to $T_{l m}^{0 t}$ or $T_{l m}^{2 e}$ or to a linear combination of them.

(3) Fixing $\xi_{m}$ allows us to cancel the components that are parallel to $T_{l m}^{2 m}$.

For now, we will use the freedom related to $\xi_{m}$ to cancel the "electric-magnetic" components, meaning that we take $h_{l m}^{\prime 2 m}=0$ for all $l$ and $m$. Understanding the gauge fixing of $\xi_{r}$ and $\xi_{e}$ that is the correct one for our purposes is not simple at this stage. Because of that, we choose to set to zero just some arbitrary linear combination of $T_{l m}^{0 t}, T_{l m}^{1 e}$, and $T_{l m}^{2 e}$ to be further determined in what follows. There is only one resulting d.o.f. that we call $h_{l}^{t e}$ that is associated 
with a linear combination of these tensors with some undetermined coefficients. More formally, we fix the gauge such that

$h_{T}=\sum_{l m} h_{l m}^{0 l} T_{l m}^{0 l}+h_{l m}^{t e}\left(\alpha T_{l m}^{0 t}+\beta T_{l m}^{1 e}+\gamma T_{l m}^{2 e}\right)+h_{l m}^{1 m} T_{l m}^{1 m}$,

where $\alpha, \beta$ and $\gamma$ are constants.

\section{Gauge fixing for the timelike components}

In order to fix the remaining gauge freedom, we will write the vector $h_{V}$ and the scalar $\xi_{S}$ as

$h_{V}=\sum_{s l m} h_{l m}^{0 s}(t, r) \bar{Y}_{l m}^{s}(\theta, \varphi), \quad \xi_{S}=\sum_{l m} \xi_{l m}^{0}(t, r) Y_{l m}(\theta, \varphi)$.
For each component of $h_{V}$ we have $h_{0 i}^{\prime}=h_{0 i}+\partial_{0} \xi_{i}+\partial_{i} \xi_{0}$ or more conveniently $h_{V}^{\prime}=h_{V}+\dot{\xi}_{V}+\nabla \xi_{S}$. By replacing with (5.11) we get

$$
\begin{aligned}
h_{V}^{\prime}= & \sum_{l m}\left(h_{l m}^{0 r}+\dot{\xi}_{l m}^{r}+\partial_{r} \xi_{l m}^{0}\right) \bar{Y}_{l m}^{r}+\left(h_{l m}^{0 e}+\dot{\xi}_{l m}^{e}+\frac{\xi_{l m}^{0}}{r}\right) \bar{Y}_{l m}^{e} \\
& +\left(h_{l m}^{0 m}+\dot{\xi}_{l m}^{m}\right) \bar{Y}_{l m}^{m} .
\end{aligned}
$$

Thus, in analogy with the case of the Maxwell field, we can fix $\xi_{0}$ in such way that $h_{l m}^{\prime 0 e}$ is zero for each $l m$, obtaining the expansion

$$
h_{V}=\sum_{l m} h_{l m}^{0 r} \bar{Y}_{l m}^{r}+h_{l m}^{0 m} \bar{Y}_{l m}^{m}
$$

\section{E. Lagrangian for each angular momentum}

The starting point is the Lagrangian (4.1). Using the decomposition of the field $h_{\mu \nu}$ given in (5.5) in terms of spatial and temporal components we obtain

$$
\begin{aligned}
\mathcal{L}= & \frac{1}{2}\left(\dot{h}_{T} \cdots \dot{h}_{T}-\operatorname{Tr}\left(\dot{h}_{T}\right) \operatorname{Tr}\left(\dot{h}_{T}\right)\right)+\frac{1}{2}\left(\nabla^{2} h_{T} \cdots h_{T}+\nabla \operatorname{Tr}\left(h_{T}\right) \cdot \nabla \operatorname{Tr}\left(h_{T}\right)\right) \\
& +\left(\nabla \cdot h_{T}\right) \cdot\left[\left(\nabla \cdot h_{T}\right)-\nabla \operatorname{Tr}\left(h_{T}\right)\right]+\nabla h_{S}\left[\left(\nabla \cdot h_{T}\right)-\nabla \operatorname{Tr}\left(h_{T}\right)\right] \\
& -2 \dot{h}_{V} \cdot\left[\left(\nabla \cdot h_{T}\right)-\nabla \operatorname{Tr}\left(h_{T}\right)\right]-\left(\nabla \cdot h_{V}\right) \cdot\left(\nabla \cdot h_{V}\right)-\nabla^{2} h_{V} \cdot h_{V} .
\end{aligned}
$$

In this matricial notation a single dot means the contraction of a one index for each tensor and two dots the contraction of the two sets of indices of the two symmetric tensors involved in the product. The full Lagrangian is given by

$$
L=\int_{\mathbb{R}}^{3} d^{3} \bar{x} \mathcal{L}=\int_{0}^{\infty} d r r^{2}\left(\int d \Omega \mathcal{L}\right) .
$$

Replacing the expressions (5.10) and (5.13) in (5.15) while considering the properties of spherical harmonics (Appendixes A and B), it turns out that we can rewrite the Lagrangian as a sum of independent modes for each $l$ and $m$

$$
L=\sum_{l m} \int_{0}^{\infty} d r\left(\mathcal{L}_{l m}^{I}+\mathcal{L}_{l m}^{I I}\right)
$$

where the $\mathcal{L}_{l m}^{I}$ contains the variables $h_{l}^{1 m}$ and $h_{l}^{0 m}$, and $\mathcal{L}_{l m}^{I I}$ involves the fields $h_{l}^{0 l}$ and $h_{l}^{\text {te }}$ together with the Lagrange multipliers $h_{l}^{0 r}$ and $h_{l}^{00}$.

The Lagrangians for the modes are independent of $m$. Again, it is clear that we will have $(2 l+1)$ equal contributions for each $l$. Accordingly, we will suppress the index $m$. After a long but straightforward calculation using the properties listed in Appendixes A and B, the Lagrangian corresponding to the mode $I$ (and $l \geq 2$ ) reads

$$
\begin{aligned}
\mathcal{L}_{l}^{I}= & \frac{r^{2}}{2} \dot{h}_{l}^{1 m} \dot{h}_{l}^{1 m}-\frac{(l-1)(l+2)}{2} h_{l}^{1 m} h_{l}^{1 m}+r^{2} \partial_{r} h_{l}^{0 m} \partial_{r} h_{l}^{0 m} \\
& +l(l+1) h_{l}^{0 m} h_{l}^{0 m}+\sqrt{2} \dot{h}_{l}^{1 m}\left(r h_{l}^{0 m}-r^{2} \partial_{r} h_{l}^{0 m}\right),
\end{aligned}
$$

and the one associated with mode II is 


$$
\begin{aligned}
\mathcal{L}_{l}^{I I}= & \frac{r^{2}}{2}\left(\beta^{2}-\alpha^{2}+\gamma^{2}\right) \dot{h}_{l}^{t e} \dot{h}_{l}^{t e}-\sqrt{2} r^{2} \alpha \dot{h}_{l}^{0 l} \dot{h}_{l}^{t e}+\frac{r^{2}}{2}\left(\alpha^{2}-\gamma^{2}\right) \partial_{r} h_{l}^{t e} \partial_{r} h_{l}^{t e} \\
& +\sqrt{2} \alpha r h_{l}^{t e} \partial_{r} h_{l}^{0 l}+h_{l}^{0 l} h_{l}^{0 l}+\left(\beta^{2}-\frac{\sqrt{l(l+1)}}{2} \alpha \beta-\frac{\sqrt{(l-1)(l+2)}}{2} \beta \gamma\right) h_{l}^{t e} h_{l}^{t e} \\
& +\sqrt{2}\left(\frac{l(l+1)}{2} \alpha-\sqrt{l(l+1)} \beta+\frac{\sqrt{(l-1) l(l+1)(l+2)}}{2} \gamma\right) h_{l}^{0 l} h_{l}^{t e}+l(l+1) h_{l}^{0 r} h_{l}^{0 r} \\
& +h_{l}^{0 r}\left[4 r \dot{h}_{l}^{0 l}-2 \sqrt{2} \alpha r^{2} \partial_{r} \dot{h}_{l}^{t e}-\sqrt{2}(2 \alpha+\sqrt{l(l+1)} \beta) r \dot{h}_{l}^{t e}\right] \\
& +h_{l}^{00}\left[-2 r \partial_{r} h_{l}^{0 l}-(l(l+1)+2) h_{l}^{0 l}+\sqrt{2} \alpha r^{2} \partial_{r} \partial_{r} h_{l}^{t e}+\sqrt{2}(3 \alpha+\sqrt{l(l+1)} \beta) r \partial_{r} h_{l}^{t e}\right. \\
& +\frac{1}{\sqrt{2}}\left(-(l-1)(l+2) \alpha+4 \sqrt{l(l+1)} \beta-\sqrt{\left.(l-1) l(l+1)(l+2) \gamma) h_{l}^{t e}\right]} .\right.
\end{aligned}
$$

In the same way, as for the case of parallel planes, we will study the modes I and II separately trying to reduce them to scalar fields for $l \geq 2$. Then, we will present the particular cases $l=0$ and $l=1$.

\section{F. Hamiltonian of mode $I$ for $\boldsymbol{l} \geq \mathbf{2}$}

From Eq. (5.17) it can be seen clearly that $h_{l}^{0 m}$ has no dynamics. Then, we get the following constraint:

$$
\begin{gathered}
-2 r^{2} \partial_{r} \partial_{r} h_{l}^{0 m}-4 r \partial_{r} h_{l}^{0 m}+2 l(l+1) h_{l}^{0 m} \\
+\sqrt{2} r^{2} \partial_{r} \dot{h}_{l}^{1 m}+3 \sqrt{2} r \dot{h}_{l}^{1 m}=0 .
\end{gathered}
$$

In an analogy with the case of the parallel planes in Eq. (5.19), this expression cannot be solved algebraically. But, the constraint can be implemented by first computing the Hamiltonian. The momenta are given by

$$
\pi_{l}^{1 m}=\frac{\partial \mathcal{L}_{l}^{I}}{\partial \dot{h}_{l}^{1 m}}=r^{2} \dot{h}_{l}^{1 m}+\sqrt{2}\left(r h_{l}^{0 m}-r^{2} \partial_{r} h_{l}^{0 m}\right) .
$$

From Eqs. (5.17) and (5.20) we compute

$$
\begin{aligned}
\mathcal{H}_{l}^{I}= & \pi_{l}^{1 m} \dot{h}_{l}^{1 m}-\mathcal{L}_{l}^{I}=\frac{\pi_{l}^{1 m} \pi_{l}^{1 m}}{2 r^{2}}+\frac{(l-1)(l+2)}{2} h_{l}^{1 m} h_{l}^{1 m} \\
& -(l-1)(l+2) h_{l}^{0 m} h_{l}^{0 m}-\sqrt{2} h_{l}^{0 m}\left(\partial_{r} \pi_{l}^{1 m}+\frac{\pi_{l}^{1 m}}{r}\right) .
\end{aligned}
$$

Now, by working with $h_{l}^{0 m}$ as a Lagrange multiplier in (5.21), the following constraint appears:

$$
-2(l-1)(l+2) h_{l}^{0 m}-\sqrt{2}\left(\partial_{r} \pi_{l}^{1 m}+\frac{\pi_{l}^{1 m}}{r}\right)=0 .
$$

Replacing (5.22) in (5.21) gives, for $l \geq 2$, the one dimensional Hamiltonian density

$$
\begin{aligned}
\mathcal{H}_{l}^{I}= & \frac{l(l+1)}{2 r^{2}} \frac{\pi_{l}^{1 m} \pi_{l}^{1 m}}{(l-1)(l+2)}+\frac{1}{2} \frac{\partial_{r} \pi_{l}^{1 m} \partial_{r} \pi_{l}^{1 m}}{(l-1)(l+2)} \\
& +\frac{1}{2}(l-1)(l+2) h_{l}^{1 m} h_{l}^{1 m},
\end{aligned}
$$

and by redefining the variables as

$\phi_{l}^{I}=\frac{\pi_{l}^{1 m}}{\sqrt{(l-1)(l+2)}}, \quad P_{l}^{I}=-\sqrt{(l-1)(l+2)} h_{l}^{1 m}$,

we reduce (5.23) to the Hamiltonian of a free scalar on the sphere

$$
\mathcal{H}_{l}^{I}=\frac{1}{2}\left(P_{l}^{I} P_{l}^{I}+\partial_{r} \phi_{l}^{I} \partial_{r} \phi_{l}^{I}+\frac{l(l+1)}{r^{2}} \phi_{l}^{I} \phi_{l}^{I}\right) .
$$

Additionally, the canonical commutation relation

$$
\left[P_{l}^{I}(t, r), \phi_{l}^{I}\left(t, r^{\prime}\right)\right]=i \delta\left(r-r^{\prime}\right)
$$

follows from the validity of

$$
\left[\pi_{l}^{1 m}(t, r), h_{l}^{1 m}\left(t, r^{\prime}\right)\right]=i \delta\left(r-r^{\prime}\right)
$$

\section{G. Hamiltonian of mode II for $\boldsymbol{l} \geq \mathbf{2}$}

For mode II, we have the Lagrangian (5.18). Working out the equations of motion of the Lagrange multiplier $h_{l}^{00}$ yields the constraint

$$
\begin{aligned}
& -2 r \partial_{r} h_{l}^{0 l}-(l(l+1)+2) h_{l}^{0 l}+\sqrt{2}(3 \alpha+\sqrt{l(l+1)} \beta) r \partial_{r} h_{l}^{t e} \\
& +\sqrt{2} \alpha r^{2} \partial_{r} \partial_{r} h_{l}^{t e}+\frac{1}{\sqrt{2}}(-(l-1)(l+2) \alpha+4 \sqrt{l(l+1)} \beta \\
& -\sqrt{(l-1) l(l+1)(l+2)} \gamma) h_{l}^{t e}=0 .
\end{aligned}
$$

Taking into account that (5.28) gives rise to nonlocal terms (that cannot be eliminated by the same means used for 
mode I), we are led to propose a particular gauge fixing such that

$$
h_{l}^{0 l}=a h_{l}^{t e}+b r \partial_{r} h_{l}^{t e},
$$

where $a$ and $b$ are constants that will be fixed to satisfy (5.28). Indeed, by replacing (5.29) in (5.28), we get

$$
\begin{aligned}
& \sqrt{2}(\alpha-\sqrt{2} b) r^{2} \partial_{r} \partial_{r} h_{l}^{t e}+(3 \sqrt{2} \alpha+\sqrt{2 l(l+1)} \beta \\
& \quad-(l(l+1)+4) b-2 a) r \partial_{r} h_{l}^{t e} \frac{1}{\sqrt{2}}(-(l-1)(l+2) \alpha \\
& \quad+4 \sqrt{l(l+1)} \beta-\sqrt{(l-1) l(l+1)(l+2)} \gamma \\
& \quad-a \sqrt{2}(l(l+1)+2)) h_{l}^{t e}=0
\end{aligned}
$$

It is possible to solve for $a, b$ and $\alpha$ in terms of $\beta$ and $\gamma$ in such a way that all the terms vanish separately. We obtain

$$
\begin{aligned}
& a=\sqrt{\frac{2}{l(l+1)}} \beta-\sqrt{\frac{(l-1)(l+2)}{2 l(l+1)} \gamma,} \\
& b=\sqrt{\frac{2}{l(l+1)}} \beta+\sqrt{\frac{2}{(l-1) l(l+1)(l+2)}} \gamma, \\
& \alpha=\frac{2}{\sqrt{l(l+1)}} \beta+\frac{2}{\sqrt{(l-1) l(l+1)(l+2)}} \gamma .
\end{aligned}
$$

It is fundamental to remark that Eq. (5.33) selects a particular gauge choice for achieving this simplification.

Replacing (5.29), (5.31), (5.32), and (5.33) in (5.18) and working with $h_{l}^{0 r}$ as a Lagrange multiplier allows us to obtain the following simple Lagrangian:

$$
\mathcal{L}_{l}^{I I}=\frac{\gamma^{2}}{2}\left[\dot{h}_{l}^{t e} \dot{h}_{l}^{t e}-\partial_{r} h_{l}^{t e} \partial_{r} h_{l}^{t e}-l(l+1) h_{l}^{t e} h_{l}^{t e}\right] .
$$

The corresponding Hamiltonian is

$$
\begin{aligned}
\mathcal{H}_{l}^{I I} & =\pi_{l}^{t e} \dot{h}_{l}^{t e}-\mathcal{L}_{l}^{I I} \\
& =\frac{1}{2}\left[\frac{\pi_{l}^{t e} \pi_{l}^{t e}}{\gamma^{2} r^{2}}+\gamma^{2} r^{2} \partial_{r} h_{l}^{t e} \partial_{r} h_{l}^{t e}+\gamma^{2} l(l+1) h_{l}^{t e} h_{l}^{t e}\right],
\end{aligned}
$$

with the canonical commutation relations

$$
\left[\pi_{l}^{t e}(t, r), h_{l}^{t e}\left(t, r^{\prime}\right)\right]=i \delta\left(r-r^{\prime}\right) .
$$

Finally, by making the identifications

$$
\phi_{l}^{I I}=\gamma r h_{l}^{t e}, \quad P_{l}^{I I}=\frac{\pi_{l}^{t e}}{r \gamma},
$$

the Hamiltonian of the scalar field modes is recovered in the form

$\mathcal{H}_{l}^{I I}=\frac{1}{2}\left[P_{l}^{I I} P_{l}^{I I}+\partial_{r} \phi_{l}^{I I} \partial_{r} \phi_{l}^{I I}+\frac{l(l+1)}{r^{2}} \phi_{l}^{I I} \phi_{l}^{I I}\right]$,

associated with the commutation relations

$$
\left[P_{l}^{I I}(t, r), \phi_{l}^{I I}\left(t, r^{\prime}\right)\right]=i \delta\left(r-r^{\prime}\right) .
$$

\section{H. Analysis of the mode $l=0$}

For the case $l=0$, the tensor spherical harmonics of spin $J=1$ and $J=2$ are not defined. Then, the Lagrangian (5.15) reduces to

$$
\begin{aligned}
\mathcal{L}_{l=0}= & \mathcal{L}_{l=0}^{I}+\mathcal{L}_{l=0}^{I I} \\
= & -\frac{r^{2}}{2} \alpha^{2} \dot{h}_{0}^{t e} \dot{h}_{0}^{t e}-\sqrt{2} r^{2} \alpha \dot{h}_{0}^{0 l} \dot{h}_{0}^{t e}+\frac{r^{2}}{2} \alpha^{2} \partial_{r} h_{0}^{t e} \partial_{r} h_{0}^{t e}+\sqrt{2} \alpha r h_{0}^{t e} \partial_{r} h_{0}^{0 l}+h_{0}^{0 l} h_{0}^{0 l}+2 \sqrt{2} h_{0}^{0 r}\left[\sqrt{2} r \dot{h}_{0}^{0 l}-\alpha r^{2} \partial_{r} \dot{h}_{0}^{t e}-\alpha r \dot{h}_{0}^{t e}\right] \\
& +\sqrt{2} h_{0}^{00}\left[-\sqrt{2} r \partial_{r} h_{l}^{0} 0 l-\sqrt{2} h_{0}^{0 l}+\alpha r^{2} \partial_{r} \partial_{r} h_{0}^{t e}+3 \alpha r \partial_{r} h_{0}^{t e}+\alpha h_{0}^{t e}\right] .
\end{aligned}
$$

The equation of motion of $h_{0}^{00}$ produces the constraint

$-\sqrt{2} r \partial_{r} h_{0}^{0 l}-\sqrt{2} h_{0}^{0 l}+\alpha r^{2} \partial_{r} \partial_{r} h_{0}^{t e}+3 \alpha r \partial_{r} h_{0}^{t e}+\alpha h_{0}^{t e}=0$.

By proposing the equivalent of (5.29) and replacing (5.41), we get that the constants $a$ and $b$ must be $a=b=$ $\alpha / \sqrt{2}$ without the need of fixing $\alpha$. We obtain

$$
h_{0}^{0 l}=\frac{\alpha}{\sqrt{2}}\left(h_{0}^{t e}+r \partial_{r} h_{0}^{t e}\right) \quad \forall \alpha .
$$

On the other hand, taking $h_{0}^{0 r}$ as a Lagrange multiplier gives

$$
\sqrt{2} r \dot{h}_{0}^{0 l}-\alpha r^{2} \partial_{r} \dot{h}_{0}^{t e}-\alpha r \dot{h}_{0}^{t e}=0 .
$$

Equations (5.42) and (5.43) are consistent with each other. Replacing both of them in (5.40) yields $\mathcal{L}_{l=0}=0$, allowing 
us to conclude that the $l=0$ mode makes no contribution to the $\mathrm{EE}$ for any choice of gauge.

\section{Analysis of the mode $l=1$}

For the case $l=1$, the tensor spherical harmonics of spin $J=0$ and $J=1$ are well defined. However, the ones corresponding to $J=2$ do not exist. Hence the Lagrangian for the mode $I$ now reads

$$
\begin{aligned}
\mathcal{L}_{l=1}^{I}= & \frac{r^{2}}{2} \dot{h}_{1}^{1 m} \dot{h}_{1}^{1 m}+r^{2} \partial_{r} h_{1}^{0 m} \partial_{r} h_{1}^{0 m}+2 h_{1}^{0 m} h_{1}^{0 m} \\
& +\sqrt{2} \dot{h}_{1}^{1 m}\left(r h_{1}^{0 m}-r^{2} \partial_{r} h_{1}^{0 m}\right) .
\end{aligned}
$$

In an analogous way to the case $l \geq 2$, we obtain $\pi_{1}^{1 m}=$ $r^{2} \dot{h}_{1}^{1 m}+\sqrt{2}\left(r h_{1}^{0 m}-r^{2} \partial_{r} h_{1}^{0 m}\right)$. Now, the Hamiltonian can be expressed as

$$
\mathcal{H}_{l=1}^{I}=\frac{\pi_{1}^{1 m} \pi_{1}^{1 m}}{2 r^{2}}-\sqrt{2} h_{1}^{0 m}\left(\partial_{r} \pi_{1}^{1 m}+\frac{\pi_{1}^{1 m}}{r}\right) .
$$

Working with $h_{1}^{0 m}$ as a Lagrange multiplier gives

$$
\mathcal{H}_{l=1}^{I}=\frac{\pi_{1}^{1 m} \pi_{1}^{1 m}}{2 r^{2}}, \quad \pi_{1}^{1 m}=r \partial_{r} \pi_{1}^{1 m} .
$$

This implies that mode I will not contribute to the EE for $l=1$.

Moreover, the Lagrangian of mode II can be given, for $l=1$, from $(5.18)$ as

$$
\begin{aligned}
\mathcal{L}_{l=1}^{I I}= & \frac{r^{2}}{2}\left(\beta^{2}-\alpha^{2}\right) \dot{h}_{1}^{t e} \dot{h}_{1}^{t e}-\sqrt{2} r^{2} \alpha \dot{h}_{1}^{0 l} \dot{h}_{1}^{t e}+\frac{r^{2}}{2} \alpha^{2} \partial_{r} h_{1}^{t e} \partial_{r} h_{1}^{t e}+\sqrt{2} \alpha r h_{1}^{t e} \partial_{r} h_{1}^{0 l}+h_{1}^{0 l} h_{1}^{0 l}+\left(\beta^{2}-\frac{\alpha \beta}{\sqrt{2}}\right) h_{1}^{t e} h_{1}^{t e} \\
& +(\sqrt{2} \alpha-2 \beta) h_{1}^{0 l} h_{1}^{t e}+2 h_{1}^{0 r} h_{1}^{0 r}+2 h_{1}^{0 r}\left[2 r \dot{h}_{1}^{0 l}-\sqrt{2} \alpha r^{2} \partial_{r} \dot{h}_{1}^{t e}-(\sqrt{2} \alpha+\beta) r \dot{h}_{1}^{t e}\right] \\
& +h_{1}^{00}\left[-2 r \partial_{r} h_{1}^{0 l}-4 h_{1}^{0 l}+\sqrt{2} \alpha r^{2} \partial_{r} \partial_{r} h_{1}^{t e}+(3 \sqrt{2} \alpha+2 \beta) r \partial_{r} h_{1}^{t e}+4 \beta h_{1}^{t e}\right] .
\end{aligned}
$$

So, $h_{1}^{00}$ yields the constraint

$$
\begin{aligned}
& -2 r \partial_{r} h_{1}^{0 l}-4 h_{1}^{0 l}+\sqrt{2} \alpha r^{2} \partial_{r} \partial_{r} h_{1}^{t e} \\
& +(3 \sqrt{2} \alpha+2 \beta) r \partial_{r} h_{1}^{t e}+4 \beta h_{1}^{t e}=0 .
\end{aligned}
$$

In this calculation, we also propose the locality relation (5.29). By replacing it in (5.48), we obtain that (for every choice of gauge) it is valid that

$$
h_{1}^{0 l}=\frac{\alpha}{\sqrt{2}} h_{1}^{t e}+r \beta \partial_{r} h_{1}^{t e} \quad \forall \alpha, \beta .
$$

Finally, using (5.49) in (5.47) produces $\mathcal{L}_{l=1}^{I I}=0$. Thus, there is no contribution of mode II for $l=1$.

\section{J. Analysis of the gauge fixing}

We have already restricted the gauge freedom with relation (5.33). This particular choice allows us to write the dynamics of the two modes in the same fashion as the one of the scalar modes. Now, we analyze if the field $h_{\mu \nu}$ or, more conveniently, the resulting d.o.f. $h_{l}^{1 m}$ and $h_{l}^{\text {te }}$ can be written in terms of gauge invariant operators inside the sphere. For this purpose, we appeal to the expression (4.3) of the gauge invariant curvature tensor.

Using a computer-based algebraic manipulation, we obtain that the mode I field given by $h_{l}^{1 m}$ can be rewritten in terms of the "electric-radial-electric-magnetic" contraction of the Riemann tensor as

$$
R_{\text {erem }}^{l m}=e^{\mu} r^{\nu} e^{\rho} m^{\sigma} R_{\mu \nu \rho \sigma}^{l m}=F_{l m}(\theta, \varphi) \frac{h_{l}^{1 m}(t, r)}{r^{2}},
$$

where $F_{l m}(\theta, \varphi)$ is a function of the angles $\theta$ and $\varphi$ for each $l$ and $m$. Specifically, for $m=0$ it is valid that

$$
\begin{aligned}
F_{l 0}(\theta)= & \frac{\pi^{\frac{5}{2}} l \sqrt{\Gamma^{3}(l+1) \Gamma(l)}}{16 \Gamma^{2}(l+2)} P_{l}^{0}(\cos \theta)\left[P_{l}^{1}(\cos \theta)\right]^{3} \\
& \times\left(4 P_{l}^{2}(\cos \theta) \cot \theta+P_{l}^{3}(\cos \theta)\right),
\end{aligned}
$$

where $P_{l}^{m}(\cos \theta)$ are the associated Legendre polynomials. The important point in this expression is that the relation between $h_{l}^{1 m}(t, r)$ and the curvature does not involve radial derivatives. That would make the algebra generated by this field nonlocal with respect to one of gauge invariant operators in the sphere.

For mode II, under the partial gauge choice (5.33), we can further set $\alpha=0$ or equivalently

$$
\gamma=-\frac{\beta}{\sqrt{(l-1)(l+2)}} .
$$

This allows us to obtain locality with respect to the curvature tensor. With this choice (5.29) reduces to an algebraic relation (without any derivatives) between the fields $h_{l}^{0 l}$ and $h_{l}^{\text {te }}$ given by

$$
h_{l}^{0 l}=\sqrt{\frac{(l-1)(l+2)}{2}} \beta h_{l}^{t e} .
$$


From this relation, it follows that the remaining field $h_{l}^{\text {te }}$ can be computed from the "electric-magnetic-electricmagnetic" contraction of the Riemann tensor in a local way in $t, r$ as

$R_{e m e m}^{l m}=e^{\mu} m^{\nu} e^{\rho} m^{\sigma} R_{\mu \nu \rho \sigma}^{l m}=G_{l m}(\theta, \varphi) \frac{h_{t e}(t, r)}{r^{2}}$,

where $G_{l m}(\theta, \varphi)$ is another function of the angles $\theta, \varphi$ for each $l$ and $m$. For $m=0$ it reads

$$
\begin{aligned}
G_{l 0}(\theta)= & \frac{\pi^{\frac{5}{2}} \beta \sqrt{l(l+2) \Gamma(l)}}{16(l+1)^{2} \sqrt{\Gamma(l+3)}}\left[P_{l}^{1}(\cos \theta)\right]^{4} \\
& \times\left(4 l(l+1) P_{l}^{0}(\cos \theta)\right. \\
& +2(l(l+1)+2) P_{l}^{1}(\cos \theta) \cot \theta \\
& \left.+(l(l+1)+2) P_{l}^{2}(\cos \theta)\right) .
\end{aligned}
$$

Therefore, by taking (5.33) and (5.52), the gauge fixed field $h_{\mu \nu}$ inside the sphere generates the same algebra as the gauge invariant operators. This algebra is equivalent to one of the modes of two scalar fields except for the $l=0,1$ modes which are absent for the helicity 2 theory.

\section{K. Entanglement entropy and logarithmic coefficient}

To sum up, the EE associated with linearized gravitons in a sphere of radius $R$ is equivalent to the one corresponding to two scalar fields without contributions of the $l=0$ and $l=1$ angular momentum modes (or a Maxwell field without the $l=1$ modes).

As we recall in Sec. III, the entanglement entropy of a scalar in a sphere has a universal logarithmic term $-1 / 90 \log (R / \epsilon)$. Also, the mode $l=0$ of the scalar corresponds to a massless $d=2$ scalar field in the $r>0$ half-line with entropy given by $1 / 6 \log (R / \epsilon)$.

To obtain the universal logarithmic term for gravitons we just need the logarithmic contribution of the $l=1$ mode for the scalar. This mode is a $d=2$ field in the half-line $r>0$ with Hamiltonian

$$
\mathcal{H}=\frac{1}{2}\left[P^{2}+\left(\partial_{r} \phi\right)^{2}+\frac{2}{r^{2}} \phi^{2}\right] .
$$

This model is scale invariant. But, in contrast with the $l=0$ mode, it contains a potential term $2 / r^{2} \phi^{2}$. We have to compute the entanglement entropy in an interval $r \in(0, R)$. The ultraviolet divergent piece of the $\mathrm{EE}$ comes from entanglement in high energy fluctuations around the boundary $r=R$. For these high energy fluctuations, the effect of the potential can be neglected. Then, we must have a divergent piece that is the same as for the usual scalar field $S \sim-1 / 6 \log (\epsilon)$. As the model does not contain any dimensionful scales, by dimensional reasons, we obtain

$$
S=\frac{1}{6} \log \left(\frac{R}{\epsilon}\right)+\text { cons. }
$$

We have checked this numerically in the lattice to an excellent (five digits) precision.

Hence, as for the $l=0$ mode, we get a $1 / 6$ coefficient for the logarithmic term of the $l=1$ modes. Consequently, we get a logarithmic coefficient for the graviton in the sphere given by twice the coefficient of the scalar subtracting two times the $l=0$ mode and $2(2 l+1)=6$ times the $l=1$ mode, obtaining

$$
2 \times\left(-\frac{1}{90}-\frac{1}{6}-3 \times \frac{1}{6}\right)=-\frac{61}{45} .
$$

As it seems to be the rule, the value of the logarithmic coefficient increases with spin. It is higher for the helicity 2 field than for Maxwell and scalar fields. The entropy on the sphere then writes

$$
S=c \frac{A}{\epsilon^{2}}-\frac{61}{45} \log \left(\frac{R}{\epsilon}\right) .
$$

\section{DISCUSSION}

We have computed the EE for free gravitons in flat space for a region between parallel planes and the sphere. For the wall, we find a universal coefficient that coincides with one of two scalar fields. For the sphere, the logarithmic term is given by $-61 / 45$, which is equivalent to two scalar fields where the $l=0$ and $l=1$ modes are missing. These results refer to clear physical quantities. First, our real-time approach allows us to clarify that these are entropies of gauge invariant operator algebras of the theory inside the regions. Second, the meaning of these universal terms for the continuum model follows from the fact that they coincide with the ones obtained using mutual information. We can write a regularized entropy as [31]

$$
S_{\epsilon}(A) \equiv \frac{1}{2} I_{\epsilon}\left(A_{+}, A_{-}\right)
$$

In this formula, one computes the mutual information between two regions $A_{+}$and $A_{-}$covering most of the inside and outside parts of the boundary of $A$, respectively, but symmetrically separated from the boundary by a distance $\epsilon / 2$. This can be thought as a form of point splitting the regularization of the entropy. The mutual information for disjoint regions is completely unambiguous in QFT and thus is $S_{\epsilon}(A)$. In particular, mutual information is unaffected by details of the algebra definition such as center terms (or edge modes). In the present case, our results for the entropy are indeed equivalent to $S_{\epsilon}(A)$. This is the case of the full scalar field EE [13] and this identification also holds for the $l=0,1$ modes. These 
later one dimensional fields have mutual information that diverges as $-1 / 3 \log (\epsilon)$ as the boundaries of $A_{+}$and $A_{-}$ approach each other. This holds for the free scalar and this UV result cannot change due to the potential or the boundary condition at the origin. ${ }^{3}$

There are other results in the literature concerning the logarithmic coefficient due to gravitons, especially in black hole backgrounds (see for example [11,12,33,34]; see also [35] and references therein for gravitons in de Sitter space). There is a general expectation that the logarithmic coefficient for the sphere should be proportional to the $A$ anomaly ${ }^{4}[8,9]$.

The free graviton does not have a symmetric gauge invariant stress tensor due to the Weinberg Witten theorem [36]. Then, the definition of the $A$ anomaly is uncertain. ${ }^{5}$ For a Maxwell field, there is a mismatch of the logarithmic term in the entanglement entropy and the $A$ anomaly which is solved by coupling the theory to (heavy) charges [16]. In the present case, a clarification of what is the right coefficient for interacting gravity seems to be further away since any interactions would take us away from the QFT setting, thus rising the problems of operator algebra localization. Eternal black holes seem to be a more natural setup in gravity than the sphere since they are related to a partition of the asymptotic space in two. In this same sense, there are also indications that in full quantum gravity a boundary separating localized d.o.f. should be an extremal surface $[1,37]$. This is, of course, the case of the entanglement wedge in holographic EE but not the sphere in Minkowski space.

A natural conjecture which presents itself from our results for the Maxwell field and the graviton is that, on the sphere, the EE of higher helicity $h>2$ fields should be equivalent to the one of two scalar fields where the $l=$ $0, \ldots, h-1$ modes are subtracted. By the same reasons discussed in the previous section, these modes have an EE given by

$$
S=\frac{1}{6} \log \left(\frac{R}{\epsilon}\right)+\mathrm{f}(1)
$$

where $f(l)$ is a function of the angular momentum. Hence, we would have a logarithmic coefficient ${ }^{6}$

\footnotetext{
${ }^{3}$ There is, however, a subleading $-1 / 2 \log (\log (R / \epsilon))$ term in the mutual information for the $l=0$ mode that is not present in the entropy (with the usual lattice regularization) [13]. This comes from superselection sectors for the $d=2$ scalar [10,32].

${ }^{4}$ For black hole backgrounds another contribution is expected proportional to the $c$ anomaly coefficient.

${ }^{5}$ We thank Sergey Solodukhin for communication regarding anomalies for the graviton.

${ }^{6}$ After this paper appeared in the arXiv database Dowker noted this same result would follow from thermodynamics in de Sitter space [38]. He also obtains the result for fermion fields of different helicity.
}

$$
-2\left(\frac{1}{90}+\frac{1}{6} \sum_{l=0}^{h-1}(2 l+1)\right)=-\frac{1+15 h^{2}}{45} .
$$

Another interesting problem is how to fix the gauge for the graviton so that $h^{\mu \nu}$ inside a region of arbitrary shape can be given in terms of the gauge invariant operators localized in the same region. We hope to come back to these problems in the future.

\section{ACKNOWLEDGMENTS}

We thank Pablo Bueno, Joan Camps, and Marina Huerta for useful discussions. This work was partially supported by CONICET, CNEA, and Universidad Nacional de Cuyo, Argentina. The work of H. C. is partially supported by an It From Qubit grant by the Simons Foundation.

\section{APPENDIX A: PROPERTIES OF VECTOR SPERICAL HARMONICS}

In this Appendix, we list some useful properties of vector spherical harmonics, some of them may also be found in $[13,28,29]$. The vector spherical harmonics are defined by (3.3), (3.4), (3.5). They satisfy the orthogonality relations

$$
\int \bar{Y}_{l m}^{s} \bar{Y}_{l m}^{s^{\prime} *} d \Omega=\delta_{s s^{\prime}} \delta_{l l^{\prime}} \delta_{m m^{\prime}}
$$

where $\bar{Y}_{l m}^{s *}$ is the complex conjugate of $\bar{Y}_{l m}^{s}$, that is also given by

$$
\bar{Y}_{l m}^{s *}=(-1)^{m} \bar{Y}_{l(-m)}^{s} .
$$

The vector spherical harmonics can be used to expand an arbitrary three component vector $\bar{V}$ as

$$
\bar{V}=\sum_{l=0}^{\infty} \sum_{m=-l}^{l} \sum_{s=r, e, m} V_{l m}^{s}(r) \bar{Y}_{l m}^{s}(\theta, \varphi)
$$

where the functions $V_{l m}^{s}(r)$ are fixed by the Fourier coefficient expression

$$
V_{l m}^{s}(r)=\int \bar{V} \cdot \bar{Y}_{l m}^{s^{*}} d \Omega
$$

The vector spherical harmonics possess the following directional properties:

$$
\hat{r} \cdot \bar{Y}_{l m}^{r}=Y_{l m}, \quad \hat{r} \cdot \bar{Y}_{l m}^{e}=0, \quad \hat{r} \cdot \bar{Y}_{l m}^{m}=0
$$

and their divergences are given by

$$
\nabla \cdot \bar{Y}_{l m}^{r}=\frac{2}{r} Y_{l m},
$$




$$
\begin{aligned}
& \nabla \cdot \bar{Y}_{l m}^{e}=-\frac{\sqrt{l(l+1)}}{r} Y_{l m}, \\
& \nabla \cdot \bar{Y}_{l m}^{m}=0 .
\end{aligned}
$$

The curls can be written as

$$
\begin{aligned}
& \nabla \times \bar{Y}_{l m}^{r}=-\frac{\sqrt{l(l+1)}}{r} \bar{Y}_{l m}^{m}, \\
& \nabla \times \bar{Y}_{l m}^{e}=\frac{1}{r} \bar{Y}_{l m}^{m}, \\
& \nabla \times \bar{Y}_{l m}^{m}=-\frac{\sqrt{l(l+1)}}{r} \bar{Y}_{l m}^{r}-\frac{1}{r} \bar{Y}_{l m}^{e} .
\end{aligned}
$$

Finally, the Laplancians can be computed to be

$$
\begin{aligned}
& \nabla^{2} \bar{Y}_{l m}^{r}=-\frac{l(l+1)+2}{r^{2}} \bar{Y}_{l m}^{r}+\frac{2 \sqrt{l(l+1)}}{r^{2}} \bar{Y}_{l m}^{e}, \\
& \nabla^{2} \bar{Y}_{l m}^{e}=\frac{2 \sqrt{l(l+1)}}{r^{2}} \bar{Y}_{l m}^{r}-\frac{l(l+1)}{r^{2}} \bar{Y}_{l m}^{e}, \\
& \nabla^{2} \bar{Y}_{l m}^{m}=-\frac{l(l+1)}{r^{2}} \bar{Y}_{l m}^{m} .
\end{aligned}
$$

\section{APPENDIX B: PROPERTIES OF TENSOR SPHERICAL HARMONICS}

In this Appendix, we list some properties of tensor spherical harmonics, some of them may also be found in $[28,29]$. They are given by Eqs. (5.2) or alternatively by the expressions (5.4). Their most useful characteristic is that they can be used as a basis for the space of symmetric tensors fields at a fixed radius. The tensor spherical harmonics satisfy the orthogonality relation

$$
\int \operatorname{Tr}\left(T_{l m}^{J S} T_{l^{\prime} m^{\prime}}^{* J^{\prime}}\right) d \Omega=\delta_{J J^{\prime}} \delta_{s s^{\prime}} \delta_{l l^{\prime}} \delta_{m m^{\prime}},
$$

where $T_{l m}^{* J S}$ is the complex conjugate of $T_{l m}^{J S}$ given by

$$
T^{* J S}=(-1)^{m} T_{l-m}^{J S} .
$$

The traces of the tensor spherical harmonics are

$$
\begin{aligned}
& \operatorname{Tr}\left(T_{l m}^{0 l}\right)=Y_{l m}, \quad \operatorname{Tr}\left(T_{l m}^{0 t}\right)=\sqrt{2} Y_{l m}, \\
& \operatorname{Tr}\left(T_{l m}^{J s}\right)=0, \quad J s=1 e, 1 m, 2 e, 2 m .
\end{aligned}
$$

They further satisfy

$$
\begin{aligned}
& \hat{r} \cdot T_{l m}^{0 l}=\bar{Y}_{l m}^{r}, \quad \hat{r} \cdot T_{l m}^{1 e}=\frac{1}{\sqrt{2}} \bar{Y}_{l m}^{e}, \quad \hat{r} \cdot T_{l m}^{2 e}=0, \\
& \hat{r} \cdot T_{l m}^{0 t}=0, \quad \hat{r} \cdot T_{l m}^{1 m}=\frac{1}{\sqrt{2}} \bar{Y}_{l m}^{m}, \quad \hat{r} \cdot T_{l m}^{2 m}=0 .
\end{aligned}
$$

The divergences of tensor spherical harmonics can be written as

$$
\nabla \cdot T_{l m}^{0 l}=\frac{2}{r} \bar{Y}_{l m}^{r}
$$

$$
\begin{aligned}
& \nabla \cdot T_{l m}^{0 t}=-\frac{\sqrt{2}}{r} \bar{Y}_{l m}^{r}+\frac{1}{r} \sqrt{\frac{l(l+1)}{2}} \bar{Y}_{l m}^{e}, \\
& \nabla \cdot T_{l m}^{1 e}=-\frac{1}{r} \sqrt{\frac{l(l+1)}{2}} \bar{Y}_{l m}^{r}+\frac{1}{r} \frac{3}{\sqrt{2}} \bar{Y}_{l m}^{e}, \\
& \nabla \cdot T_{l m}^{1 m}=\frac{1}{r} \frac{3}{\sqrt{2}} \bar{Y}_{l m}^{m},
\end{aligned}
$$

$$
\begin{aligned}
& \nabla \cdot T_{l m}^{2 e}=-\frac{1}{r} \sqrt{\frac{(l-1)(l+2)}{2}} \bar{Y}_{l m}^{e}, \\
& \nabla \cdot T_{l m}^{2 m}=-\frac{1}{r} \sqrt{\frac{(l-1)(l+2)}{2}} \bar{Y}_{l m}^{m},
\end{aligned}
$$

and the Laplacians are the following:

$$
\begin{gathered}
\nabla^{2} T_{l m}^{0 l}=-\frac{l(l+1)+4}{r^{2}} T_{l m}^{0 l}+\frac{2 \sqrt{2}}{r^{2}} T_{l m}^{0 t} \\
+\frac{2 \sqrt{2 l(l+1)}}{r^{2}} T_{l m}^{1 e}, \\
\nabla^{2} T_{l m}^{0 t}=\frac{2 \sqrt{2}}{r^{2}} T_{l m}^{0 l}-\frac{l(l+1)+2}{r^{2}} T_{l m}^{0 t}-\frac{2 \sqrt{l(l+1)}}{r^{2}} T_{l m}^{l e}, \\
\nabla^{2} T_{l m}^{1 e}=\frac{2 \sqrt{2 l(l+1)}}{r^{2}} T_{l m}^{0 l}-\frac{2 \sqrt{l(l+1)}}{r^{2}} T_{l m}^{0 t} \\
\nabla^{2} T_{l m}^{1 e}+\frac{2 \sqrt{(l-1)(l+2)}}{r^{2}} T_{l m}^{2 e}, \\
\nabla^{2} T_{l m}^{1 m}=-\frac{l(l+1)+4}{r^{2}} T_{l m}^{1 m}+\frac{2 \sqrt{(l-1)(l+2)}}{r^{2}} T_{l m}^{2 m}, \\
\nabla^{2} T_{l m}^{2 e}=\frac{2 \sqrt{(l-1)(l+2)}}{r^{2}} T_{l m}^{1 e}-\frac{(l-1)(l+2)}{r^{2}} T_{l m}^{2 e}, \\
\nabla_{l m}^{2 m}=
\end{gathered}
$$


[1] J. Camps, Superselection sectors of gravitational subregions, J. High Energy Phys. 01 (2019) 182.

[2] W. Donnelly and S. B. Giddings, How is quantum information localized in gravity?, Phys. Rev. D 96, 086013 (2017).

[3] S. Ryu and T. Takayanagi, Holographic Derivation of Entanglement Entropy from AdS/CFT, Phys. Rev. Lett. 96, 181602 (2006).

[4] T. Faulkner, A. Lewkowycz, and J. Maldacena, Quantum corrections to holographic entanglement entropy, J. High Energy Phys. 11 (2013) 074.

[5] B. Czech, J. L. Karczmarek, F. Nogueira, and M. Van Raamsdonk, The gravity dual of a density matrix, Classical Quantum Gravity 29, 155009 (2012).

[6] D. L. Jafferis, A. Lewkowycz, J. Maldacena, and S. J. Suh, Relative entropy equals bulk relative entropy, J. High Energy Phys. 06 (2016) 004.

[7] X. Dong, D. Harlow, and A. C. Wall, Reconstruction of Bulk Operators within the Entanglement Wedge in GaugeGravity Duality, Phys. Rev. Lett. 117, 021601 (2016).

[8] S. N. Solodukhin, Entanglement entropy, conformal invariance and extrinsic geometry, Phys. Lett. B 665, 305 (2008).

[9] H. Casini, M. Huerta, and R. C. Myers, Towards a derivation of holographic entanglement entropy, J. High Energy Phys. 05 (2011) 036.

[10] H. Casini, M. Huerta, J. M. Magán, and D. Pontello, Entanglement entropy and superselection sectors. I. Global symmetries, arXiv:1905.10487.

[11] S. N. Solodukhin, Entanglement entropy of black holes, Living Rev. Relativity 14, 8 (2011).

[12] A. Sen, Logarithmic corrections to Schwarzschild and other non-extremal black hole entropy in different dimensions, J. High Energy Phys. 04 (2013) 156.

[13] H. Casini and M. Huerta, Entanglement entropy of a Maxwell field on the sphere, Phys. Rev. D 93, 105031 (2016).

[14] J. S. Dowker, Entanglement entropy for even spheres, arXiv: 1009.3854 .

[15] K. W. Huang, Central charge and entangled gauge fields, Phys. Rev. D 92, 025010 (2015).

[16] H. Casini, M. Huerta, J. M. Magan, and D. Pontello, On the logarithmic coefficient of the entanglement entropy of a Maxwell field, arXiv:1911.00529.

[17] W. Donnelly and A. C. Wall, Geometric entropy and edge modes of the electromagnetic field, Phys. Rev. D 94, 104053 (2016).

[18] S. Ghosh, R. M. Soni, and S. P. Trivedi, On the entanglement entropy for gauge theories, J. High Energy Phys. 09 (2015) 069.
[19] H. Casini, M. Huerta, and J. A. Rosabal, Remarks on entanglement entropy for gauge fields, Phys. Rev. D 89, 085012 (2014).

[20] H. Casini and M. Huerta, Entanglement and alpha entropies for a massive scalar field in two dimensions, J. Stat. Mech. (2005) P12012.

[21] H. Casini and M. Huerta, Entanglement entropy in free quantum field theory, J. Phys. A 42, 504007 (2009).

[22] M. Srednicki, Entropy and Area, Phys. Rev. Lett. 71, 666 (1993).

[23] H. Casini and M. Huerta, Entanglement entropy for the n-sphere, Phys. Lett. B 694, 167 (2010).

[24] R. Lohmayer, H. Neuberger, A. Schwimmer, and S. Theisen, Numerical determination of entanglement entropy for a sphere, Phys. Lett. B 685, 222 (2010).

[25] P. Calabrese and J. L. Cardy, Entanglement entropy and quantum field theory, J. Stat. Mech. (2004) P06002.

[26] T. Ortin, Gravity and Strings (Cambridge University Press, Cambridge, England, 2010).

[27] C. W. Misner, K. S. Thorne, and J. A. Wheeler, Gravitation (W. H. Freeman and Company, San Francisco, 1973), p. 1279.

[28] G. Compère, R. Oliveri, and A. Seraj, Gravitational multipole moments from Noether charges, J. High Energy Phys. 05 (2018) 054.

[29] K. S. Thorne, Multipole expansions of gravitational radiation, Rev. Mod. Phys. 52, 299 (1980).

[30] T. Regge and J. A. Wheeler, Stability of a Schwarzschild singularity, Phys. Rev. 108, 1063 (1957).

[31] H. Casini, M. Huerta, R. C. Myers, and A. Yale, Mutual information and the F-theorem, J. High Energy Phys. 10 (2015) 003.

[32] R. E. Arias, H. Casini, M. Huerta, and D. Pontello, Entropy and modular Hamiltonian for a free chiral scalar in two intervals, Phys. Rev. D 98, 125008 (2018).

[33] D. V. Fursaev and G. Miele, Cones, spins and heat kernels, Nucl. Phys. B484, 697 (1997).

[34] S. N. Solodukhin, Newton constant, contact terms and entropy, Phys. Rev. D 91, 084028 (2015).

[35] D. V. Vassilevich, One loop quantum gravity on de Sitter space, Int. J. Mod. Phys. A 08, 1637 (1993).

[36] S. Weinberg and E. Witten, Limits on massless particles, Phys. Lett. 96B, 59 (1980).

[37] J. Camps, The parts of the gravitational field, arXiv: 1905.10121.

[38] J. S. Dowker, Note on the entanglement entropy of higher spins in four dimensions, arXiv:1908.04870. 